\title{
Reactions of $\mathrm{MoCl}_{5}$ and $\mathrm{MoO}_{2} \mathrm{Cl}_{2}$ with Succinimide, 1,4-Diaminobutane, 3-Methylpyridine, 1,3-Diaminopropane, Pyrazole and 1-Methylpyrrolidine in Tetrahydrofuran
}

\author{
RAKESH KUMAR and GURSHARAN SINGH* \\ Research Scholar registered with Punjab Technical University, Kapurthala, India. \\ Department of Applied Chemistry, Giani Zail Singh Campus College of \\ Engineering \& Technology, Dabwali Road, MRSPTU Bathinda-151001, India. \\ *Corresponding author E-mail: gursharans82@gmail.com \\ http://dx.doi.org/10.13005/ojc/370306
}

(Received: May 04, 2021; Accepted: June 05, 2021)

\begin{abstract}
It has been reported that molybdenum may extract oxygen from oxygen containing ligands. Oxo complexes of above bases with transition metals show numerous applications and are biologically active. So to study the biological activity of molybdenum complexes and to study oxo abstraction reactions by molybdenum, reactions of succinimide/1,4-diaminobutane/ 3-methylpyridine/1, 3-diaminopropane/pyrazole/1-methylpyrrolidine with $\mathrm{MoCl}_{5} / \mathrm{MoO}_{2} \mathrm{Cl}_{2}$ have been carried out, in THF medium using equimolar/bimolar quantities of the ligand, at normal temperature. The products thus obtained are: $\mathrm{Mo}_{2} \mathrm{O}_{3} \mathrm{Cl}_{5}\left(\mathrm{C}_{4} \mathrm{H}_{5} \mathrm{NO}_{2}\right)_{2}\left(\mathrm{C}_{4} \mathrm{H}_{8} \mathrm{O}\right)_{2},[1] ; \mathrm{Mo}_{2} \mathrm{O}_{2} \mathrm{Cl}_{2}\left(\mathrm{C}_{4} \mathrm{H}_{5} \mathrm{NO}_{2}\right)_{2}\left(\mathrm{C}_{4} \mathrm{H}_{8} \mathrm{O}\right)_{2}$, [2]; $\mathrm{MoO}_{2} \mathrm{Cl}_{2}\left(\mathrm{HNCH}_{2} \mathrm{CH}_{2} \mathrm{CH}_{2} \mathrm{CH}_{2} \mathrm{NH}_{2}\right)_{2},[3] ; \mathrm{Mo}_{3} \mathrm{Cl}_{8}\left(\mathrm{C}_{6} \mathrm{H}_{7} \mathrm{~N}\right)_{4}\left(\mathrm{C}_{4} \mathrm{H}_{8} \mathrm{O}\right)$, [4]; $\mathrm{Mo}_{3} \mathrm{Cl}_{6}\left(\mathrm{C}_{6} \mathrm{H}_{7} \mathrm{~N}\right)_{6}\left(\mathrm{C}_{4} \mathrm{H}_{8} \mathrm{O}\right)_{6},[5]$; $\mathrm{MoO}_{2} \mathrm{Cl}_{3}\left(\mathrm{HNCH}_{2} \mathrm{CH}_{2} \mathrm{CH}_{2} \mathrm{NH}_{2}\right)_{2},[6] ; \mathrm{Mo}_{2} \mathrm{O}_{4} \mathrm{Cl}_{4}\left(\mathrm{C}_{3} \mathrm{H}_{4} \mathrm{~N}_{2}\right)_{4}$, [7] and $\mathrm{Mo}_{2} \mathrm{O}_{6} \mathrm{Cl}_{8}\left(\mathrm{C}_{5} \mathrm{H}_{11} \mathrm{~N}\right)_{4}$, [8]. There is oxygen abstraction by molybdenum during the reaction from the oxygen containing solvent THF. Formulations of these compounds were made and their properties were studied with FTIR (transmission mode), ${ }^{1} \mathrm{H}$ NMR/ $/{ }^{13} \mathrm{C}$ NMR, microbiological studies, elemental analysis (Mo, $\mathrm{Cl}, \mathrm{C}, \mathrm{H}, \mathrm{N}$ ) and LC-MS. All preparations, separations and isolations were executed in vacuum line and inert atmosphere (dry nitrogen) to eliminate any oxidation/hydrolysis of products by air/moisture. The formulations proposed have been supported by the above characterization studies.
\end{abstract}

Keywords: Succinimide, 1, 4-diaminobutane, 3-methylpyridine, 1, 3-diaminopropane, pyrazole, 1-methylpyrrolidine.

\section{INTRODUCTION}

\section{Succinimide}

Succinimides ${ }^{1}$ are involved in various biological applications. Succinimide is a constituent of various biologically active compounds having significance as: CNS depressant ${ }^{2}$, hypotensive ${ }^{3}$, antitumour ${ }^{4}$, cytostatic ${ }^{5}$, bacteriostatic $^{6}$, nerve conduction blocking ${ }^{7}$, muscle relaxant ${ }^{8}$, anorectic ${ }^{9}$, antibacterial $^{10}$, analgesic ${ }^{11}$, anti-convulsant ${ }^{12}$, antitubercular ${ }^{13}$, antispasmodic ${ }^{14}$ and antifungal ${ }^{15}$.

Succinimide can form complex through oxygen atom. Deprotonated succinimide (on removal of hydrogen from nitrogen) can form complex through the nitrogen atom. In deprotonated succinimide, the

This is an Open Access article licensed under a Creative Commons license: Attribution 4.0 International (CC- BY). Published by Oriental Scientific Publishing Company @ 2018

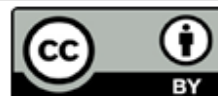


$\mathrm{CN}$ bond length lies between single and double bond, because the new free electron pair of nitrogen gets delocalized and is spread on both $\mathrm{CN}$ bonds due to conjugation between lone pair of nitrogen and $\pi$-electrons of $\mathrm{C}=\mathrm{O}$ bonds ${ }^{16}$. This conjugation reduces chances of availability of this lone pair for coordination. So coordination in succinimide through oxygen is more likely.

\section{1, 4-Diaminobutane}

1, 4-Diaminobutane ${ }^{17-19}$ has a variety of applications in agrochemicals, paint additives, pharmaceuticals, surfactants and micronutrients. It is used as starting material in some biological systems and amido-ureas. It is used in the preparation of nylon 46. It acts as corrosion inhibitor of mild steel in $1 \mathrm{~N} \mathrm{H}_{2} \mathrm{SO}_{4}$.

\section{3-Methylpyridine}

3-Methylpyridine 20 has application in agrochemical industry as chlorpyrifos ${ }^{21}$. 3-Methylpyridine degrades/evaporates slowly from water samples as compared to 2-methylpyridine/4methylpyridine ${ }^{22,23}$. 3-Methylpyridine is precursor to prepare antidote for organophosphate poisoning ${ }^{24}$. It is used as waterproofing agent ${ }^{25}$ in textile industry.

\section{1,3-Diaminopropane}

1, 3-Diaminopropane ${ }^{26}$ has a variety of industrial applications, such as, in epoxy resin and cross-linking agents. It is used as precursor for preparation of pharmaceuticals, organic chemicals and agrochemicals. It is used ${ }^{27}$ in the synthesis of heterocycles used in coordination complexes and textile finishing. It is a potential inhibitor ${ }^{28,29}$ against neoplasia and ornithine decarboxylase enzyme protein on rat urinary bladder carcinogenesis.

\section{Pyrazole}

Pyrazoles as well as their derivatives have a lot of biological properties, like antipshycotic ${ }^{30-32}$, antimicrobial $^{33}$, anticonvulsant ${ }^{34}$, antitumor ${ }^{35}$, anticyclooxygenase $^{36}$, analgesic ${ }^{37}$, antitubercular ${ }^{38}$, antidiabetic $^{39}$, antiinflammatory ${ }^{40}$, etc.

\section{1-Methylpyrrolidine}

1-Methylpyrrolidine ${ }^{41}$ has numerous applications in agrochemicals, pharmaceuticals, colourants, organic synthesis, photographic chemicals, plasticizers, emulsifiers, rubber chemicals, curing agent for epoxy resins, corrosion inhibitors, etc. It is used in PU manufacture as a catalyst. In cosmetics, it acts as a powerful surfactant. It is present in cigarette smoke. It is part of cefepime $^{42}$ : broad-spectrum cephalosporin antibiotic capable to treat bacteria causing pneumonia, infections of the skin and urinary tract.

Transition metals complexes on coordination with ligands undergo deep change in physiological properties of the metals and ligands. Desired properties ${ }^{27,43}$ on transition metals for particular applications can be incorporated by coordination of metals with certain ligands. It involves modification of properties, like stability of oxidation states, electrophilic/nucleophilic properties and solvophilicity of the metal ions. We have to choose a suitable metal and the ligand after various trials. On coordination, metal and ligand properties undergo a desired change. Many drugs containing metal chelates have higher biological activity than the uncoordinated ligands themselves ${ }^{44-49}$.

\section{Aim of Investigation}

$\mathrm{MoCl}_{5}$ reacts with a variety of bases. The author has investigated ${ }^{50-55}$ the reactions of $\mathrm{MoCl}_{5}$ with 4-phenylimidazole-2-thiol, aromatic azoles, imides, diaminoalkanes, alkylpyridines, 2-thiazoline2-thiol, thiols and mercaptopyridine- $\mathrm{N}$-oxide sodium. $\mathrm{MoO}_{2} \mathrm{Cl}_{2}$ reacts with various bases. The author has investigated ${ }^{50-58}$ the reactions of $\mathrm{MoO}_{2} \mathrm{Cl}_{2}$ with aromatic azoles, alkanediols, diaminoalkanes, imides, amides, thioamides, purine and thiols.

It has been reported that molybdenum may extract ${ }^{59,60}$ oxygen from oxygen containing ligands. Oxo complexes of above bases with transition metals show numerous applications and are biologically active. So to study the biological activity of molybdenum complexes and to study oxo abstraction reactions by molybdenum, reactions of succinimide/1,4diaminobutane/3-methylpyridine/1,3-diaminopropane/ pyrazole/1-methylpyrrolidine with $\mathrm{MoCl}_{5} / \mathrm{MoO}_{2} \mathrm{Cl}_{2}$ have been carried out. Formulations of these compounds were made and their properties were studied with FTIR, ${ }^{1} \mathrm{H} N M R /{ }^{13} \mathrm{C}$ NMR, microbiological studies, elemental analysis and LC-MS.

\section{MATERIALS AND METHODS}

Succinimide, 1,4-diaminobutane, 3-methylpyridine, 1,3-diaminopropane, pyrazole, 
1-methylpyrrolidine, $\mathrm{MoCl}_{5}$ and $\mathrm{MoO}_{2} \mathrm{Cl}_{2}$ were procured from Sigma-Aldrich.

The reactants and products are sensitive to air/moisture, so all preparations, separations and isolations were executed in vacuum line and dry atmosphere (dry nitrogen) to eliminate any oxidation/ hydrolysis of reactants/products by air/moisture.

Ligand solution in dry THF was dropped from dropping funnel with continuous agitation to $\mathrm{MoCl}_{5} / \mathrm{MoO}_{2} \mathrm{Cl}_{2}$ solution in THF taken in $100 \mathrm{~mL}$ round bottom flask. The reaction was carried out for about 7 hours. The products were isolated after filtration through filtration unit fitted with G-4 sintered glass crucible.

Oxinate gravimetric method ${ }^{61}$ was used for molybdenum estimation. Mixture of sodium carbonate and sodium peroxide was fused with a known weight of the sample by using nickel crucible. Contents were fused in muffle furnace for 1 hours. at $400^{\circ} \mathrm{C}$. Fused mixture was extracted with distilled water and content was filtered through fine filter paper. Discarded the residue and only filtrate was retained. Added methyl red indicator to the filtrate. $2 \mathrm{~N}$ sulphuric acid was added to it dropwise to make it acidic. Added $2 \mathrm{~N}$ ammonium acetate solution dropwise until colour of solution became faint. Solution was heated to boiling. Added 3\% oxine solution (in glacial acetic acid) dropwise until yellow precipitates obtained. Boiled gently with stirring until the precipitation was complete. Filtered the yellow precipitate with G-4 sintered glass crucible. Precipitate were washed with hot water, dried at $130-140^{\circ} \mathrm{C}$ and weighed as $\mathrm{MoO}_{2}\left(\mathrm{C}_{9} \mathrm{H}_{6} \mathrm{NO}\right)_{2}$.

Estimation of chlorine was carried out gravimetrically as silver chloride ${ }^{61}$. A known weight of the sample was taken in distilled water. Added 10-12 pallets of sodium hydroxide in it. Content was boiled, cooled and filtered through a fine filter paper. Acidified the solution with dilute nitric acid. Added excess of $\mathrm{N} / 10$ aqueous solution of $\mathrm{AgNO}_{3}$ until white precipitate of $\mathrm{AgCl}$ were obtained. Boiled the solution until precipitation and coagulation were complete. Filtered the precipitate through G-4 sintered glass crucible, washed with acetone, dried at $130-140^{\circ} \mathrm{C}$ and weighed as $\mathrm{AgCl}$.

Carbon, hydrogen and nitrogen were estimated by Thermo Finnigan Elemental Analyser. Perkin-Elmer 400 FTIR Spectrometer was used for obtaining infrared spectra (transmission mode). Multinuclear Brucker Avance-II 400 NMR spectrometer was used for recording ${ }^{1} \mathrm{H} /{ }^{13} \mathrm{C}$ NMR in DMSO- $d_{6}$ solvent. LC-MS spectra in the range $0-1100 \mathrm{~m} / \mathrm{z}$ have been attained. These studies were executed in SAIF at P. U. Chandigarh.

Molybdenum compounds prepared were tested using agar well diffusion assay method for their antibacterial and antifungal potential on the strains: Staphylococcus aureus (Gram-positive bacteria) (MTCC-737), E. coli (Gram-negative bacteria) (MTCC-1687), Candida albicans (fungus) (MTCC-227) and Aspergillus niger (fungus) (MTCC282). Standard drugs amoxicillin and ketoconazole were used for bacteria and virus, respectively as reference. Cultures of MTCC (The Microbial Type Culture Collection and Gene Bank, Chandigarh, India) were used. Drug testing at ISF Analytical Laboratory (ISF College of Pharmacy), Ferozepur Road, Moga, Punjab (India) was carried out. R (Residue)/ F (Filtrate) refer to product source.

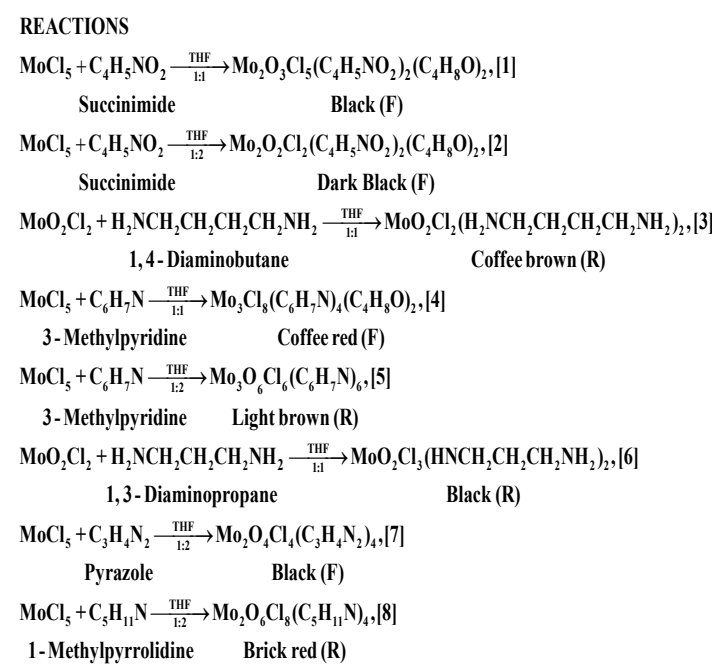

\section{RESULTS AND DISCUSSIONS}

\section{Elemental Estimation}

Percentage of the observed (theoretical) values of the elements has been depicted in Table-1. 
Table 1: Analytical data of Mo halide derivatives with succinimide

\begin{tabular}{|c|c|c|c|c|c|}
\hline Compounds & $\mathrm{Cl}$ & Mo & $\mathrm{H}$ & $\mathrm{C}$ & $\mathrm{N}$ \\
\hline $\begin{array}{l}\mathrm{Mo}_{2} \mathrm{O}_{3} \mathrm{Cl}_{5}\left(\mathrm{C}_{4} \mathrm{H}_{5} \mathrm{NO}_{2}\right)_{2}\left(\mathrm{C}_{4} \mathrm{H}_{8} \mathrm{O}\right)_{2},[1] \\
(\text { Black/759.5) }\end{array}$ & $\begin{array}{c}22.87 \\
(23.37)\end{array}$ & $\begin{array}{c}24.73 \\
(25.27)\end{array}$ & $\begin{array}{c}3.23 \\
(3.42)\end{array}$ & $\begin{array}{c}24.67 \\
(25.27)\end{array}$ & $\begin{array}{c}4.13 \\
(3.68)\end{array}$ \\
\hline $\begin{array}{l}\mathrm{Mo}_{2} \mathrm{O}_{2} \mathrm{Cl}_{2}\left(\mathrm{C}_{4} \mathrm{H}_{5} \mathrm{NO}_{2}\right)_{2}\left(\mathrm{C}_{4} \mathrm{H}_{8} \mathrm{O}\right)_{2},[2] \\
(\text { Dark black/637.0) }\end{array}$ & $\begin{array}{c}11.55 \\
(11.14)\end{array}$ & $\begin{array}{c}29.42 \\
(30.14)\end{array}$ & $\begin{array}{c}3.68 \\
(4.08)\end{array}$ & $\begin{array}{c}29.87 \\
(30.14)\end{array}$ & $\begin{array}{c}4.74 \\
(4.39)\end{array}$ \\
\hline $\begin{array}{l}\mathrm{Mo}_{2} \mathrm{O}_{2} \mathrm{Cl}_{2}\left(\mathrm{H}_{2} \mathrm{NCH}_{2} \mathrm{CH}_{2} \mathrm{CH}_{2} \mathrm{CH}_{2} \mathrm{NH}_{2}\right)_{2},[3] \\
\text { (Coffee brown/375.0) }\end{array}$ & $\begin{array}{c}18.23 \\
(18.93)\end{array}$ & $\begin{array}{l}24.73 \\
(25.6)\end{array}$ & $\begin{array}{l}6.48 \\
(6.4)\end{array}$ & $\begin{array}{l}24.79 \\
(25.6)\end{array}$ & $\begin{array}{c}14.27 \\
(14.93)\end{array}$ \\
\hline $\begin{array}{l}\mathrm{Mo}_{3} \mathrm{Cl}_{8}\left(\mathrm{C}_{6} \mathrm{H}_{7} \mathrm{~N}\right)_{4}\left(\mathrm{C}_{4} \mathrm{H}_{8} \mathrm{O}\right)_{2},[4] \\
\text { (Coffee red/1088.0) }\end{array}$ & $\begin{array}{l}25.33 \\
(26.1)\end{array}$ & $\begin{array}{c}25.69 \\
(26.47)\end{array}$ & $\begin{array}{c}3.39 \\
(4.04)\end{array}$ & $\begin{array}{c}34.67 \\
(35.29)\end{array}$ & $\begin{array}{c}4.64 \\
(5.14)\end{array}$ \\
\hline $\begin{array}{l}\mathrm{Mo}_{3} \mathrm{O}_{6} \mathrm{Cl}_{6}\left(\mathrm{C}_{6} \mathrm{H}_{7} \mathrm{~N}\right)_{6},[5] \\
\text { (Light brown/1055.0) }\end{array}$ & $\begin{array}{c}17.73 \\
(18.44)\end{array}$ & $\begin{array}{c}24.69 \\
(24.93)\end{array}$ & $\begin{array}{c}3.93 \\
(3.63)\end{array}$ & $\begin{array}{l}36.78 \\
(37.4)\end{array}$ & $\begin{array}{c}6.98 \\
(7.27)\end{array}$ \\
\hline $\begin{array}{l}\mathrm{MoO}_{2} \mathrm{Cl}_{3}\left(\mathrm{HNCH}_{2} \mathrm{CH}_{2} \mathrm{CH}_{2} \mathrm{NH}_{2}\right)_{2},[6] \\
(\text { Black/380.5) }\end{array}$ & $\begin{array}{c}27.02 \\
(27.98)\end{array}$ & $\begin{array}{c}24.67 \\
(25.22)\end{array}$ & $\begin{array}{c}5.13 \\
(4.73)\end{array}$ & $\begin{array}{c}17.98 \\
(18.92)\end{array}$ & $\begin{array}{r}14.13 \\
(14.71)\end{array}$ \\
\hline $\begin{array}{l}\mathrm{Mo}_{2} \mathrm{O}_{4} \mathrm{Cl}_{4}\left(\mathrm{C}_{3} \mathrm{H}_{4} \mathrm{~N}_{2}\right)_{4},[7] \\
(\text { Black/670.0) }\end{array}$ & $\begin{array}{c}20.68 \\
(21.19)\end{array}$ & $\begin{array}{c}27.93 \\
(28.65)\end{array}$ & $\begin{array}{c}2.71 \\
(2.38)\end{array}$ & $\begin{array}{c}20.98 \\
(21.49)\end{array}$ & $\begin{array}{c}16.04 \\
(16.71)\end{array}$ \\
\hline $\begin{array}{l}\mathrm{Mo}_{2} \mathrm{O}_{6} \mathrm{Cl}_{8}\left(\mathrm{C}_{5} \mathrm{H}_{11} \mathrm{~N}\right)_{4},[8] \\
(\text { Brick red/912.0) }\end{array}$ & $\begin{array}{c}30.47 \\
(31.14)\end{array}$ & $\begin{array}{c}20.28 \\
(21.05)\end{array}$ & $\begin{array}{c}5.36 \\
(4.82)\end{array}$ & $\begin{array}{c}27.11 \\
(26.31)\end{array}$ & $\begin{array}{c}5.84 \\
(6.14)\end{array}$ \\
\hline
\end{tabular}

\section{FTIR Spectra}

$\mathrm{N}-\mathrm{H}$ stretching of succinimide ${ }^{62,63}$ have been noted at $3409 \mathrm{~cm}^{-1} \& 3221 \mathrm{~cm}^{-1}$. Strong absorption at $3294 \mathrm{~cm}^{-1}$ indicates that [1] contains $\mathrm{N}-\mathrm{H}$ group. Bands at $980 \mathrm{~cm}^{-1}$ and $919 \mathrm{~cm}^{-1}$ support the availability of cis- $\mathrm{MoO}_{2}{ }^{2+}$ core ${ }^{64,65}$ in [1]. Occurrence of cis- $\mathrm{MoO}_{2}{ }^{2+}$ core is due to oxo abstraction ${ }^{59,60}$ by molybdenum from THF. There is some decrease in $\mathrm{C}=\mathrm{O}$ sym and asym absorptions. There is decrease in $\mathrm{C}=\mathrm{O}$ bond order, referring to the presence of
$\mathrm{O} \rightarrow$ Mo coordination ${ }^{16}$ in [1] (Table 2).

Strong absorption at $3433 \mathrm{~cm}^{-1}$ indicates that [2] contains $\mathrm{N}-\mathrm{H}$ group. Bands at $984 \mathrm{~cm}^{-1}$ and $923 \mathrm{~cm}^{-1}$ support the availability of cis- $\mathrm{MoO}_{2}{ }^{2+}$ core ${ }^{64,65}$ in [2]. Occurrence of cis- $\mathrm{MoO}_{2}{ }^{2+}$ core is due to oxo abstraction ${ }^{59,60}$ by molybdenum from THF. There is some decrease in $\mathrm{C}=\mathrm{O}$ sym and asym absorptions. There is decrease in $\mathrm{C}=\mathrm{O}$ bond order, referring to the presence of $\mathrm{O} \rightarrow$ Mo coordination ${ }^{16}$ in [2] (Table-2).

Table 2: (FTIR absorptions in $\mathrm{cm}^{-1}$ )

\begin{tabular}{|c|c|c|c|}
\hline Assignments & Succinimide ${ }^{62,63}$ & {$[1]$} & {$[2]$} \\
\hline $\mathrm{N}-\mathrm{H}$ str. & $3409 \mathrm{sb}, 3221 \mathrm{sb}$ & $\begin{array}{l}3509.6 \mathrm{sh}, 3381.5 \mathrm{sh} \\
3294.4 \mathrm{~s}, 3152.4 \mathrm{~s}, \mathrm{~b}\end{array}$ & $3433.5 \mathrm{sb}$ \\
\hline $\mathrm{CH}_{2}$ sym. str. & 2960,2947 w & $2983.7 \mathrm{sh}$ & \\
\hline $\mathrm{C}=\mathrm{O}$ sym., $\mathrm{H}-\mathrm{N}-\mathrm{C}$ in plane bending & $1774 \mathrm{~m}$ & $1776.9 \mathrm{~m}, 1756.0 \mathrm{~m}$ & $1772.8 \mathrm{w}$ \\
\hline $\mathrm{C}=\mathrm{O}$ asym., $\mathrm{H}-\mathrm{N}-\mathrm{C}$ in plane bending & 1710 vs, b & $1686.0 \mathrm{~s} 1705.2 \mathrm{~s}$ & $1637.2 \mathrm{~m}$ \\
\hline $\mathrm{CH}_{2}$ sym. scissoring & $1430 \mathrm{~m}$ & $1415.1 \mathrm{sh}$ & \\
\hline $\mathrm{CH}_{2}$ asym. scissoring & $1401 \mathrm{~m}$ & $1399.1 \mathrm{sh}$ & $1399.8 \mathrm{sh}$ \\
\hline $\mathrm{C}-\mathrm{N}-\mathrm{C}$ asym. str., $\mathrm{H}-\mathrm{N}-\mathrm{C}$ in plane bending & $1347 \mathrm{~s}, 1337$ & $1373.7 \mathrm{~m}, 1358.9 \mathrm{sh}$ & $1373.3 \mathrm{w}$ \\
\hline $\mathrm{CH}_{2}$ bending, ring in plane bending & $1298 \mathrm{~s}$ & $1297.9 \mathrm{~s}$ & $1298.1 \mathrm{w}$ \\
\hline $\mathrm{CH}_{2}$ bending & 1240 & $1248.1 \mathrm{~m}$ & $1247.1 \mathrm{sh}$ \\
\hline $\mathrm{C}-\mathrm{N}-\mathrm{C}$ asym. str., $\mathrm{H}-\mathrm{N}-\mathrm{C}$ in plane bending & $1189 \mathrm{~s}$, & $1183.2 \mathrm{~s}$ & $1189.9 \mathrm{~m}$ \\
\hline C-C str., CNC sym. str. & 849 & $857.2 \mathrm{w}$ & \\
\hline $\mathrm{CH}_{2}$ bending, ring out of plane bending & $820 \mathrm{~s}$ & $817.1 \mathrm{~s}$ & \\
\hline OCN asym. out of plane bending & $632 \mathrm{~m}$ & $645.9 \mathrm{~s}$ & $642.4 \mathrm{~m}$ \\
\hline $\begin{array}{l}\text { OCN sym. out of plane bending, } \mathrm{CH}_{2} \text { bending } \\
\text { Mo-N str. }\end{array}$ & $539 w$ & $552.2 \mathrm{w}$ & $\begin{array}{l}563.2 \mathrm{~m} \\
418.0 \mathrm{sh}\end{array}$ \\
\hline $\mathrm{Mo}=\mathrm{O}$ str. of cis- $\mathrm{MoO}_{2}{ }^{2+}$ core $\mathrm{e}^{64,65}$ & & $\begin{array}{l}980.6 \mathrm{~s} \\
919.1 \mathrm{w}\end{array}$ & $\begin{array}{l}984.7 \mathrm{w}, \\
923.5 \mathrm{sh}\end{array}$ \\
\hline
\end{tabular}

$\mathrm{N}-\mathrm{H}$ stretching of 1,4-diaminobutane ${ }^{66}$ has been detected at $3345 \mathrm{~cm}^{-1} \& 3278 \mathrm{~cm}^{-1}$. Strong N-H absorptions have been recorded at $3391 \mathrm{~cm}^{-1}, 3077 \mathrm{~cm}^{-1}$ and $3010 \mathrm{~cm}^{-1}$ in [3] (Table 3). Terminal $\mathrm{Mo}=\mathrm{O}$ stretching occurs ${ }^{67}$ at $990 \mathrm{~cm}^{-1}$
$-1010 \mathrm{~cm}^{-1}$ in various inert solvents. A medium $\mathrm{Mo}=\mathrm{O}$ stretching ${ }^{64,67,68}$ at $921 \mathrm{~cm}^{-1}$ conforms to the presence of terminal $\mathrm{Mo}=\mathrm{O}$ group. There is a decline in $\mathrm{Mo}=\mathrm{O}$ stretching to $921 \mathrm{~cm}^{-1}$ showing Mo coordination ${ }^{69}$ to 1,4-diaminobutane through $\mathrm{N}$ 
atom, in a direction trans to $\mathrm{Mo}=\mathrm{O}$ bond. Bending mode due to $\mathrm{NH}_{2}$ observed in 1,4-diaminobutane ${ }^{66}$ at $1146 \mathrm{~cm}^{-1}$ is declined to $1116 \mathrm{~cm}^{-1}$, because of $\mathrm{N} \rightarrow$ Mo coordination.

Table 3: (FTIR absorptions in $\mathrm{cm}^{-1}$ )

\begin{tabular}{lcc}
\hline Assignments & 1,4-Diaminobutane ${ }^{66}$ & [3] \\
\hline $\mathrm{N}-\mathrm{H}$ str. & 3345,3278 & $3391.2 \mathrm{~s}, 3077.1 \mathrm{~s}, 3010.1 \mathrm{~s}$ \\
$\mathrm{CH}_{2}$ str. & $2961-2874$ & $2881.1 \mathrm{sh}$ \\
$\mathrm{NH}_{2}$ bending & 1608 & $1614.2 \mathrm{~s}$ \\
$\mathrm{CH}_{2}$ deformation (strong) & $1498,1391,1355,1310$ & $1519.3 \mathrm{~m}, 1470.7 \mathrm{~m}, 1448.1 \mathrm{~s}, 1403.3 \mathrm{w}, 1344.4 \mathrm{w}$ \\
$\mathrm{NH}_{2}$ bending & 1146 & $1184.4 \mathrm{w}, 1116.2 \mathrm{~s}$ \\
$\mathrm{C}-\mathrm{N}$ sym str. (weak) & 1071 & $1025.3 \mathrm{~m}$ \\
$\mathrm{CH}_{2}$ deformation (medium) & 862,736 & $817.1 \mathrm{~s}$ \\
Mo-N (strong) & & $498.3 \mathrm{~m}$ \\
Terminal Mo=O & & $921.2 \mathrm{~m}$ \\
\hline
\end{tabular}

3-methylpyridine ${ }^{70-73}$ shows ring $\mathrm{C}-\mathrm{H}$ absorptions at $3060 \mathrm{~cm}^{-1}$ and $3032 \mathrm{~cm}^{-1}$. Strong bands at $3119 \mathrm{~cm}^{-1}$ and $3054 \mathrm{~cm}^{-1}$ have been noticed in [4]. Ring $\mathrm{C}=\mathrm{N}$ stretching \& ring $\mathrm{C}=\mathrm{N}$ torsion wave numbers have increased and ring $\mathrm{C}-\mathrm{H}$ bending mode wave numbers have declined due to $\mathrm{Mo}(\mathrm{d} \pi) \rightarrow \mathrm{N}(\mathrm{p} \pi)$ back bonding. A strong band at $989 \mathrm{~cm}^{-1}$ reveals the presence of terminal $\mathrm{Mo}=\mathrm{O}^{64,67,68}$ group in [4] (Table 4).
Strong band at $3391 \mathrm{~cm}^{-1}$ has been noticed in [5]. Ring $\mathrm{C}=\mathrm{N}$ stretching \& ring $\mathrm{C}=\mathrm{N}$ torsion wave numbers have increased and ring $\mathrm{C}-\mathrm{H}$ bending mode wave numbers have declined due to $\mathrm{Mo}(\mathrm{d} \pi) \rightarrow \mathrm{N}(\mathrm{p} \pi)$ back bonding. A medium band at $989 \mathrm{~cm}^{-1}$ reveals the presence of terminal $\mathrm{Mo}=\mathrm{O}^{64,67,68}$ group in [5] (Table 4). Occurrence of terminal $\mathrm{Mo}=\mathrm{O}$ is due to oxo abstraction ${ }^{59,60}$ by molybdenum from THF.

Table 4: (FTIR absorptions in $\mathrm{cm}^{-1}$ )

\begin{tabular}{|c|c|c|c|}
\hline Assignments & 3-Methylpyridine ${ }^{70-73}$ & {$[4]$} & {$[5]$} \\
\hline $\mathrm{C}-\mathrm{H}$ ring str. & 3060,3032 & $3168.9 \mathrm{~s}, 3119.9 \mathrm{~s}, 3054.8 \mathrm{~s}$ & $3391.0 \mathrm{~s} \mathrm{~b}$ \\
\hline $\mathrm{C}-\mathrm{H}$ methyl str. & $3002,2958,2928$ & $2937.5 \mathrm{sh}, 2866.8 \mathrm{sh}$ & \\
\hline Ring str. & 1600,1578 & $1629.1 \mathrm{~m}, 1607.1 \mathrm{~m}, 1543.1 \mathrm{~s}$ & $1630.7 \mathrm{~s}, 1554.6 \mathrm{~s}$ \\
\hline Ring $\mathrm{C}-\mathrm{H}$ bending & 1478 & & \\
\hline $\mathrm{C}-\mathrm{H}$ methyl assym bending & 1458,1452 & $1469.1 \mathrm{~m}$ & $1474.2 \mathrm{~m}$ \\
\hline Ring $\mathrm{C}-\mathrm{H}$ bending & 1415 & & \\
\hline $\mathrm{C}-\mathrm{H}$ methyl sym. bending & 1388 & $1387.1 \mathrm{w}$ & $1386.2 \mathrm{w}$ \\
\hline Ring $\mathrm{C}-\mathrm{H}$ bending & 1361 & $1306.2 \mathrm{w}$ & \\
\hline Ring str. & 1250 & $1258.1 \mathrm{w}$ & $1264.3 \mathrm{w}$ \\
\hline $\mathrm{C}-\mathrm{C}$ bond between ring and methyl str. & 1228 & & \\
\hline Ring $\mathrm{C}-\mathrm{H}$ bending & 1190 & $1180.2 \mathrm{w}$ & $1186.2 \mathrm{sh}$ \\
\hline $\mathrm{C}-\mathrm{H}$ methyl rocking & 1127,1044 & $1116.9 \mathrm{~s}$ & $1119.2 \mathrm{w}$ \\
\hline Ring out of plane bending & 1030 & $1044.1 \mathrm{sh}, 1019.1 \mathrm{sh}$ & $1048.5 \mathrm{w}$ \\
\hline Ring $\mathrm{C}=\mathrm{N}$ str. & 792 & 883.1 vs, $785.7 \mathrm{~s}, 738.7 \mathrm{~m}$ & $891.1 \mathrm{~s}, 788.1 \mathrm{~s}$ \\
\hline Ring $\mathrm{C}=\mathrm{N}$ torsion & 710 & $722.7 \mathrm{~m}$ & $721.9 \mathrm{~m}$ \\
\hline Ring bending & 635,540 & $676.6 \mathrm{~s}, 511.1 \mathrm{~m}$ & $678.0 \mathrm{~s}, 630.3 \mathrm{sh}, 568.4 \mathrm{sh}, 513.8 \mathrm{sh}$ \\
\hline$\partial \mathrm{C}-\mathrm{C}$ bond between ring and methyl & 458 & $463.1 \mathrm{~m}$ & $463.3 w$ \\
\hline Terminal $\mathrm{Mo}=\mathrm{O}^{64,67,68}$ str. & & $989.9 \mathrm{~s}$ & $949.9 \mathrm{~m}$ \\
\hline
\end{tabular}

1,3-Diaminopropane ${ }^{74}$ shows $\mathrm{N}-\mathrm{H}$ absorption bands in the range $3052-3351 \mathrm{~cm}^{-1}$. Absorptions at $3427 \mathrm{~cm}^{-1} \& 3014 \mathrm{~cm}^{-1}$ suggest the presence of $\mathrm{N}-\mathrm{H}$ group in [6] (Table 5). A strong band at $944 \mathrm{~cm}^{-1}$ is attributed to terminal $\mathrm{Mo}=\mathrm{O}^{64,67,68}$ stretching. $\mathrm{NH}_{2}$ bending absorption in the range $1159 \mathrm{~cm}^{-1}-1182 \mathrm{~cm}^{-1}$ is also shifted to lower wave number $1108 \mathrm{~cm}^{-1}$, mainly due to coordination with molybdenum. Occurrence of terminal $\mathrm{Mo}=\mathrm{O}$ is due to oxo abstraction ${ }^{59,60}$ by molybdenum from THF.

Table 5: (FTIR absorptions in $\mathrm{cm}^{-1}$ )

\begin{tabular}{|c|c|c|}
\hline Absorptions & 1, 3-Diaminopropane ${ }^{74}$ & {$[6]$} \\
\hline $\mathrm{N}-\mathrm{H}$ str. & $3052-3351$ & $3427.1 \mathrm{~m}, 3014.1 \mathrm{v} \mathrm{s}$ \\
\hline $\mathrm{CH}_{2}$ str. & $2873-2961$ & $2898.3 \mathrm{sh}, 2787.7 \mathrm{sh}, 2701.1 \mathrm{sh}$ \\
\hline $\mathrm{NH}_{2}$ bending & 1627 & $1609.1 \mathrm{~s}$ \\
\hline $\mathrm{CH}_{2}$ deformation & $1571-1582$ & $1481.1 \mathrm{~m}, 1464.5 \mathrm{~s}, 1409.1$ \\
\hline $\mathrm{NH}_{2}$ bending & $1159-1182$ & $1193.2 \mathrm{~m}, 1108.2 \mathrm{~m}$ \\
\hline C-N sym str. & 1062 & $1031.3 \mathrm{sh}$ \\
\hline $\begin{array}{l}\mathrm{CH}_{2} \text { deformation } \\
\mathrm{Mo}-\mathrm{N}\end{array}$ & 742,841 & $\begin{array}{c}887.1 \mathrm{~s}, 786.1 \mathrm{~m} \\
449.3 \mathrm{w}\end{array}$ \\
\hline Terminal $\mathrm{Mo}=\mathrm{O}^{64,67,68}$ str. & & $944.9 \mathrm{~s}$ \\
\hline
\end{tabular}


$\mathrm{N}-\mathrm{H}$ stretching of pyrazole ${ }^{75,76}$ occurs at $3452 \mathrm{~cm}^{-1}$. Band at $3386 \mathrm{~cm}^{-1}$ reflects that [7] contains $\mathrm{N}-\mathrm{H}$ group. $\mathrm{N}-\mathrm{H}$ stretching is declined due to $\mathrm{N} \rightarrow$ Mo coordination. Medium band at 969 $\mathrm{cm}^{-1}$ shows the existence of terminal $\mathrm{Mo}=\mathrm{O}^{64,67,68}$ in [7] (Table 6). Occurrence of terminal $\mathrm{Mo}=\mathrm{O}$ is due to oxo abstraction ${ }^{59,60}$ by molybdenum from tetrahydrofuran.

Table 6: (FTIR absorptions in $\mathrm{cm}^{-1}$ )

\begin{tabular}{lcc}
\hline Absorptions & Pyrazole $^{75,76}$ & {$[7]$} \\
\hline $\mathrm{N}-\mathrm{H}$, str. & 3452 & $3386.9 \mathrm{~s}$ \\
$\mathrm{C}-\mathrm{H}$ str. & 3153,3142 & $3149.9 \mathrm{sh}$ \\
$\mathrm{C}=\mathrm{C}$ ring str. & 1560 & $1631.0 \mathrm{~m}, 1519.0 \mathrm{sh}$ \\
$\mathrm{N}-\mathrm{C}$ ring str. & 1467,1140 & $1474.5 \mathrm{w}, 1402.2 \mathrm{sh}, 1350.3 \mathrm{w}$ \\
$\mathrm{C}-\mathrm{H}$ in plane bending & 1047,1037 & $1126.0 \mathrm{w}, 1052.2 \mathrm{w}$ \\
$\mathrm{C}-\mathrm{H}$ out of plane bending (wagging), ring twisting & $891,841,762$ & $777.0 \mathrm{~m}, 760.1 \mathrm{sh}, 740.8 \mathrm{sh}$ \\
Ring twisting & 658,620 & $675.4 \mathrm{sh}, 605.2 \mathrm{sh}, 581.8 \mathrm{sh}$ \\
Mo-N & & $437.7 \mathrm{sh}$ \\
Ring twisting, $\mathrm{N}-\mathrm{H}$ wagging & 501 & $518.7 \mathrm{w}$ \\
Terminal Mo=O ${ }^{64,67,68}$ str. & & $969.6 \mathrm{~m}$ \\
\hline
\end{tabular}

1-Methylpyrrolidine ${ }^{77-79}$ shows strong $\mathrm{C}-\mathrm{H}$ symmetric stretching at $2971 \mathrm{~cm}^{-1}$ and $\mathrm{C}-\mathrm{H}$ asymmetric stretching at $2890 \mathrm{~cm}^{-1}, 2832 \mathrm{~cm}^{-1}$, $2780 \mathrm{~cm}^{-1}$. C-H asymmetric stretching of [8] is found at $2750 \mathrm{~cm}^{-1}$. Weak band corresponding to the presence of terminal $\mathrm{Mo}=\mathrm{O}^{64,67,68}$ str. is observed at $976 \mathrm{~cm}^{-1}$ in [8] (Table 7). A peak at $3410 \mathrm{~cm}^{-1}$ may be due to 1-methylpyrrolidinium cation. Occurrence of terminal $\mathrm{Mo}=\mathrm{O}$ is due to oxo abstraction ${ }^{61,62}$ by molybdenum from tetrahydrofuran.

Table 7: (FTIR absorptions in $\mathrm{cm}^{-1}$ )

\begin{tabular}{ccc}
\hline Absorptions & 1-Methylpyrrolodine ${ }^{77-79}$ & {$[8]$} \\
\hline $\mathrm{N}+-\mathrm{H}$ & & $3410.6 \mathrm{v} \mathrm{s}$, \\
$\mathrm{v}_{\mathrm{s}} \mathrm{C}-\mathrm{H}$ sym. str. & $2971 \mathrm{~s}$ & \\
$\mathrm{v}_{\text {as }} \mathrm{C}-\mathrm{H}$ asym. sym. str. & $2890 \mathrm{sh}, 2832 \mathrm{~m}, 2780 \mathrm{~s}$ & $2750.3 \mathrm{sh}$ \\
$(\mathrm{C}-\mathrm{H})$ deformation & $1450 \mathrm{~s}$ & $1634.0 \mathrm{~m}, 1462.0 \mathrm{w}$ \\
$\mathrm{v}(\mathrm{C}-\mathrm{C})$ str. & $1364 \mathrm{~s}$ & $1383.7 \mathrm{sh}$ \\
$\mathrm{U}(\mathrm{C}-\mathrm{N})$ str. & $1245 \mathrm{~s}, 1202 \mathrm{~m}$ & $1163 \mathrm{~s}, 1113 \mathrm{~m}, 1108.6 \mathrm{w}$ \\
$(\mathrm{C}-\mathrm{H})$ bending & $1046 \mathrm{~s}$ & $1004.6 \mathrm{sh}$ \\
$\mathrm{CH} \mathrm{H}_{2}$ rocking & $877 \mathrm{~s}$ & $734.9 \mathrm{~m} \mathrm{~b}$ \\
$\mathrm{CNC}$ deformation & $575 \mathrm{w}$ & \\
Terminal Mo=O $\mathrm{O}^{64,67,68}$ str. & & $976.1 \mathrm{w}$ \\
\hline
\end{tabular}

\section{${ }^{1} \mathrm{H}$ NMR Spectra}

Spectra were taken in DMSO- $d_{6}$ solvent. Solvent residual peak of DMSO- $d_{6}{ }^{80}$ occurs at 2.50 ppm. THF ${ }^{80}$ spectrum in DMSO- $\mathrm{d}_{6}$ shows $\mathrm{O}-\mathrm{CH}_{2}$ peak and $\mathrm{CH}_{2}$ peak at $3.60 \mathrm{ppm}$ and $1.76 \mathrm{ppm}$, respectively. In the spectra given below, $\uparrow$ and $\downarrow$ represent upfield/downfield shift.

Succinimide ${ }^{51,53} \mathrm{CH}_{2}$ absorb at 2.73 ppm. Spectrum of [1] in DMSO-d $\mathrm{d}_{6}$ shows $\mathrm{CH}_{2}$ absorption at 3.63 ppm showing downfield shift due to decrease in electron density around these protons on coordination with molybdenum through carbonyl group (Fig.1, Table 8).

Spectrum of [2] in DMSO- $\mathrm{d}_{6}$ shows $\mathrm{CH}_{2}$ absorption at $3.42 \mathrm{ppm}$ showing downfield shift due to decrease in electron density around these protons on coordination with molybdenum through carbonyl group (Fig. 2, Table 8).

Table 8: ( ${ }^{1} \mathrm{H}$ NMR absorptions in ppm)

\begin{tabular}{cccc}
\hline Assignments & $\begin{array}{c}\text { Succinimide } \\
\text { in } \mathrm{CDCl}_{3}\end{array}$ & [1] & [2] \\
\hline $\mathrm{N}-\mathrm{H}$ & 8.9 & $11.06 \downarrow$ & $10.97 \downarrow$ \\
$\mathrm{CH}_{2}$ & 2.73 & $3.63 \downarrow$ & $3.42 \downarrow$ \\
Residua $^{80}$ DMSO-d $_{6}$ & & 2.54 & 2.48 \\
\hline
\end{tabular}

1,4-Diaminobutane ${ }^{81}$ in $\mathrm{H}_{2} \mathrm{O}$ shows $\mathrm{N}-\mathrm{H}$ peak at $1.15 \mathrm{ppm}$. NMR of [3] in DMSO- $\mathrm{d}_{6}$ suggests that $\mathrm{NH}_{2}$ peak has shifted downfield. Peak of side $\mathrm{CH}_{2}$ (attached to $\mathrm{N}$ which coordinates) as well as peak of middle $\mathrm{CH}_{2}$ (attached to outer $\mathrm{CH}_{2}$ on the side in which $\mathrm{N}$ coordinates) have shifted down field due to decrease in electron density around these protons on $\mathrm{N} \rightarrow$ Mo coordination (Fig. 3, Table 9). 
Table 9: ('H NMR absorptions in ppm)

\begin{tabular}{lcc}
\hline Assignments & 1, 4-Diaminobutane ${ }^{81}$ in $\mathrm{H}_{2} \mathrm{O}$ & [3] \\
\hline $\mathrm{NH}_{2}$ & $1.144 \mathrm{H}$ & $8.23 \downarrow$ \\
Side $\mathrm{CH}_{2}$ (attached to $\mathrm{N}$ which coordinates) & $3.02-3.054 \mathrm{H}$ & $3.52 \downarrow$ \\
Middle $\mathrm{CH}_{2}$ (attached to outer $\mathrm{CH}_{2}$ on the side in which $\mathrm{N}$ coordinates) & $1.75-1.784 \mathrm{H}$ & $2.66 \downarrow$ \\
Residual ${ }^{80}$ DMSO- $\mathrm{d}_{6}$ & & 2.43 \\
\hline
\end{tabular}

Comparison of 3-methylpyridine ${ }^{71,72,82}$ spectrum with that of [4], shows that there is downfield shift for all protons. This is due to reduction in ring $\pi$-electron density around these protons on sharing of lone pair by nitrogen with molybdenum (Fig. 4, Table 10).
Further, in the spectrum of [5], it is found that there is downfield shift for all protons. This is due to reduction in ring $\pi$-electron density around these protons on sharing of lone pair by nitrogen with molybdenum (Fig. 5, Table 10).

Table 10: ('H NMR absorptions in ppm)

\begin{tabular}{|c|c|c|c|}
\hline Absorptions & 3-Methylpyridine ${ }^{71,72,82}$ in $\mathrm{CDCl}_{3}$ & {$[4]$} & {$[5]$} \\
\hline $\mathrm{H}\left(\mathrm{CH}_{3}\right)$ & 2.32 3H Singlet & $3.27 \downarrow$ & $2.56 \downarrow$ \\
\hline $\mathrm{H}-\mathrm{C}_{1}$ & $8.441 \mathrm{H}$ Singlet & $8.77 \downarrow$ & $8.80 \downarrow$ \\
\hline $\mathrm{H}-\mathrm{C}_{3}$ & $7.451 \mathrm{H}$ Doublet & $8.40 \downarrow$ & $8.23 \downarrow$ \\
\hline $\mathrm{H}-\mathrm{C}_{4}$ & $7.161 \mathrm{H}$ Triplet & $7.92 \downarrow$ & $7.82 \downarrow$ \\
\hline $\mathrm{H}-\mathrm{C}_{5}$ & $8.421 \mathrm{H}$ Doublet & $8.70 \downarrow$ & $8.74 \downarrow$ \\
\hline Residual $^{80}$ DMSO- $_{6}$ & & 2.46 & 2.49 \\
\hline $\mathrm{THF}^{80} \mathrm{C}-2,5$ (attached to $\mathrm{N}$ ) & & 3.53 & \\
\hline $\mathrm{THF}^{80} \mathrm{C}-3,4$ & & 1.69 & \\
\hline
\end{tabular}

Comparison of spectrum of 1, 3-diaminopropane $^{83}$ with that of [6] (Fig. 6, Table 11) in DMSO- $\mathrm{d}_{6}$ suggests that $\mathrm{NH}_{2}$ and $\mathrm{CH}_{2}$ absorptions of 1,3-diaminopropane have downfield shift. This is because of decrease in electron density around these protons on $\mathrm{N} \rightarrow$ Mo coordination.

Table 11: ( ${ }^{1} \mathrm{H}$ NMR absorptions in ppm)

\begin{tabular}{lcc}
\hline Assignments & $\begin{array}{c}\text { 1, 3-Diaminopropane } \\
\text { in } \mathrm{CDCl}_{3}\end{array}$ & [6] \\
\hline $\mathrm{NH}_{2}{\mathrm{Middle} \mathrm{CH}_{2}}_{\mathrm{M}_{2}}$ & $1.214 \mathrm{H}$ & $7.89 \downarrow$ \\
Side $\mathrm{CH}_{2}$ (attached to N & $1.592 \mathrm{H}$ & $2.87 \downarrow$ \\
which coordinates) & $2.764 \mathrm{H}$ & $3.56 \downarrow$ \\
Residual & & \\
\hline
\end{tabular}

Spectrum of pyrazole ${ }^{72,84}$ in $\mathrm{CCl}_{4}$ shows absorptions due to middle $\mathrm{C}-\mathrm{H}$ proton at $6.31 \mathrm{ppm}$, $\mathrm{C}-\mathrm{H}$ protons on other two carbons at $7.61 \mathrm{ppm}$ and due to $\mathrm{N}-\mathrm{H}$ proton at $12.64 \mathrm{ppm}$. Spectrum of [7] shows that all the pyrazole $\mathrm{CH}$ protons have moved downfield (Fig. 7, Table 12). This is because of decrease in electron density around these protons on $\mathrm{N} \rightarrow$ Mo coordination. Due to keto-enol tautomerization equilibrium, peaks of $\mathrm{CH}$ protons of pyrazole appear as singlets.

Table 12: ( ${ }^{\mathrm{H}} \mathrm{H}$ NR absorptions in ppm)

\begin{tabular}{|c|c|c|}
\hline Assignments & Pyrazole $^{72,84}$ in $\mathrm{CCl}_{4}$ & [7] \\
\hline $\mathrm{N}-\mathrm{H}$ & $12.641 \mathrm{H}$ & $9.42 \uparrow$ \\
\hline Middle $\mathrm{CH}$ & $6.31(\mathrm{~s}) 1 \mathrm{H}$ & $6.51 \uparrow$ \\
\hline Side $\mathrm{CH}$ & $7.61(\mathrm{~s}) 2 \mathrm{H}$ & $7.96 \uparrow$ \\
\hline Residual ${ }^{80}$ DMSO- $_{6}$ & & 2.57 \\
\hline
\end{tabular}

Spectrum of [8] shows that all the $\mathrm{CH}$ absorptions of 1-methylpyrrolidine ${ }^{77}$ have moved downfield, referring to decline in electron density of the ring on $\mathrm{N} \rightarrow$ Mo coordination (Fig. 8, Table 13). There is a broad peak at $11.10 \mathrm{ppm}$ indicating formation of 1-methylpyrrolidinium ion.

Table 13: ( ${ }^{1} \mathrm{H}$ NMR absorptions in ppm)

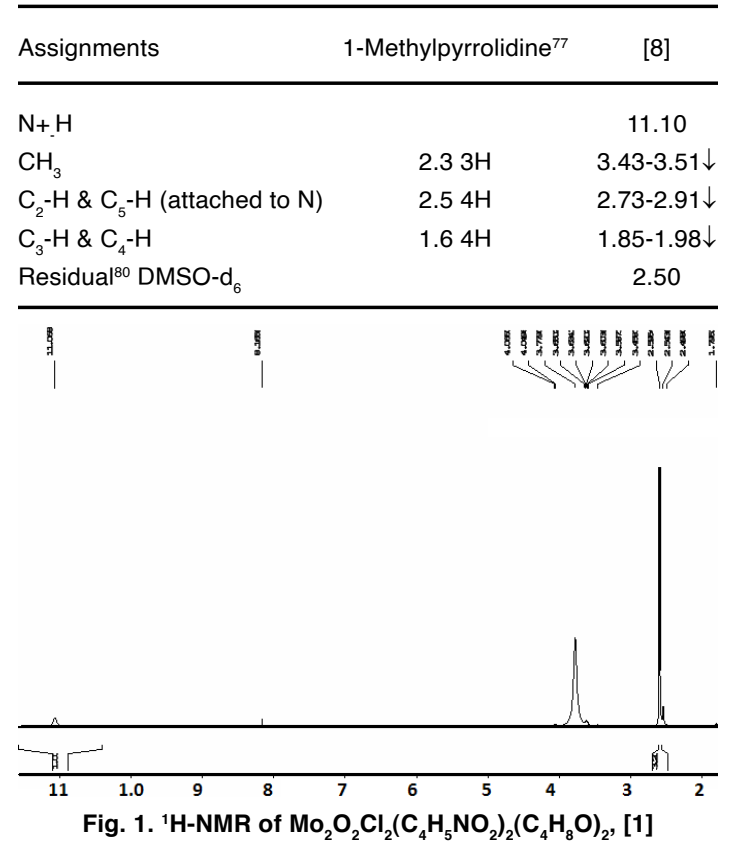




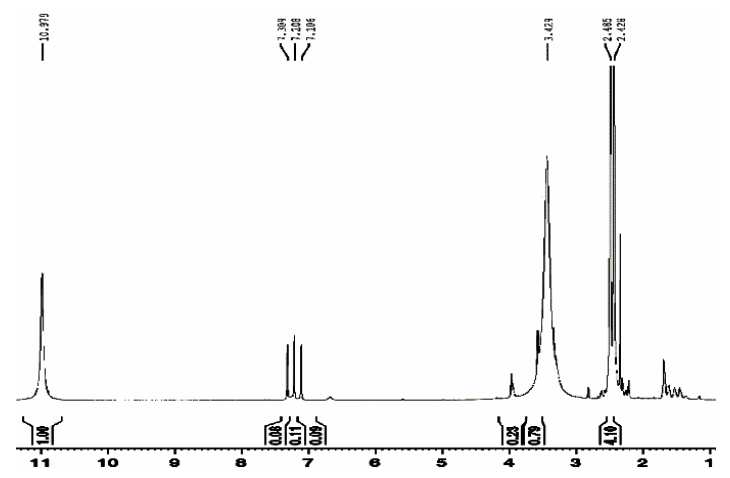

Fig. 2. ${ }^{1} \mathrm{H}-\mathrm{NMR}$ of $\mathrm{MO}_{2} \mathrm{O}_{3} \mathrm{Cl}_{5}\left(\mathrm{C}_{4} \mathrm{H}_{5} \mathrm{NO}_{2}\right)_{2}\left(\mathrm{C}_{4} \mathrm{H}_{8} \mathrm{O}\right)_{2}$, [2]

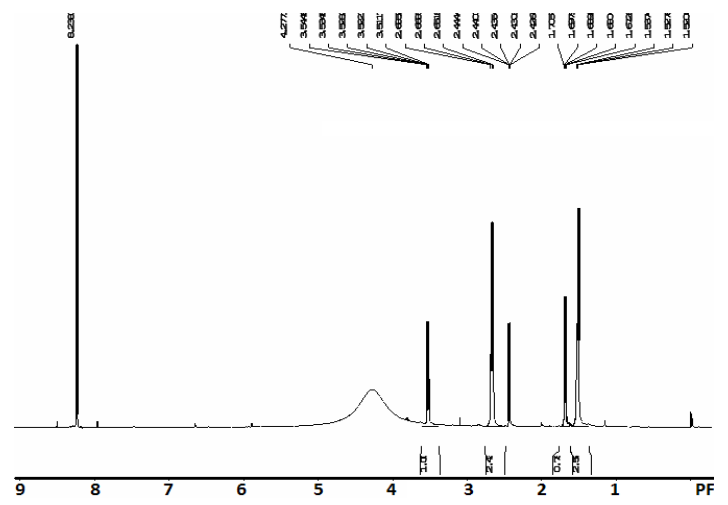

Fig. 3. ${ }^{1} \mathrm{H}-\mathrm{NMR}$ of $\mathrm{MO}_{2} \mathrm{O}_{2} \mathrm{Cl}_{2}\left(\mathrm{H}_{2} \mathrm{NCH}_{2} \mathrm{CH}_{2} \mathrm{CH}_{2} \mathrm{CH}_{2} \mathrm{NH}_{2}\right)_{2}$, [3]
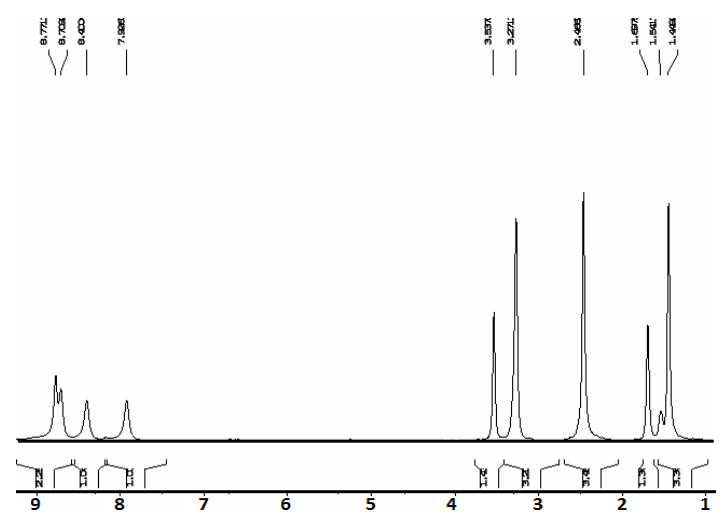

Fig. 4. ${ }^{1} \mathrm{H}-\mathrm{NMR}$ of $\mathrm{Mo}_{3} \mathrm{Cl}_{8}\left(\mathrm{C}_{6} \mathrm{H}_{7} \mathrm{~N}\right)_{4}\left(\mathrm{C}_{4} \mathrm{H}_{8} \mathrm{O}\right)_{2}$, [4]
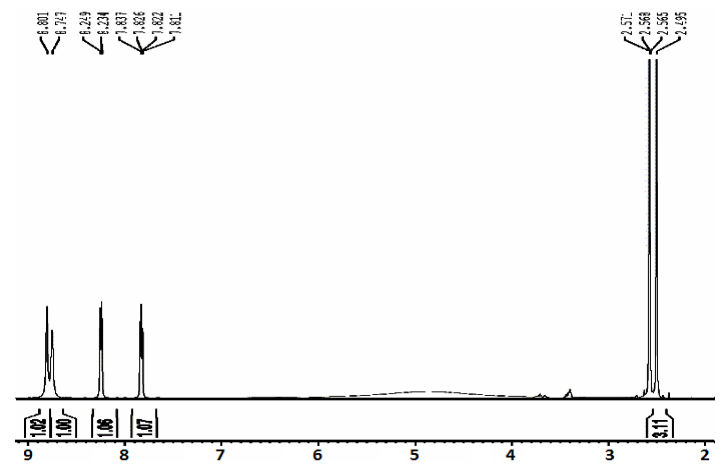

Fig. 5: ' $\mathrm{H}-\mathrm{NMR}$ of $\mathrm{Mo}_{3} \mathrm{O}_{6} \mathrm{Cl}_{6}\left(\mathrm{C}_{6} \mathrm{H}_{7} \mathrm{~N}\right)_{6}$, [5]

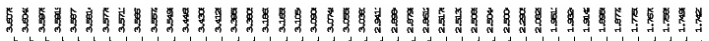
(N)

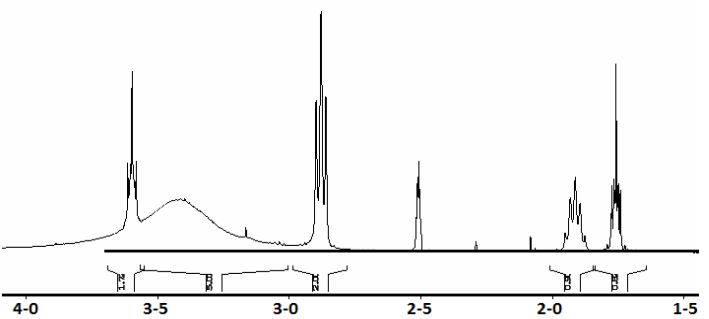

Fig. 6. ' $\mathrm{H}-\mathrm{NMR}$ of $\mathrm{MoO}_{2} \mathrm{Cl}_{3}\left(\mathrm{HNCH}_{2} \mathrm{CH}_{2} \mathrm{CH}_{2} \mathrm{NH}_{2}\right)_{2}$, [6]

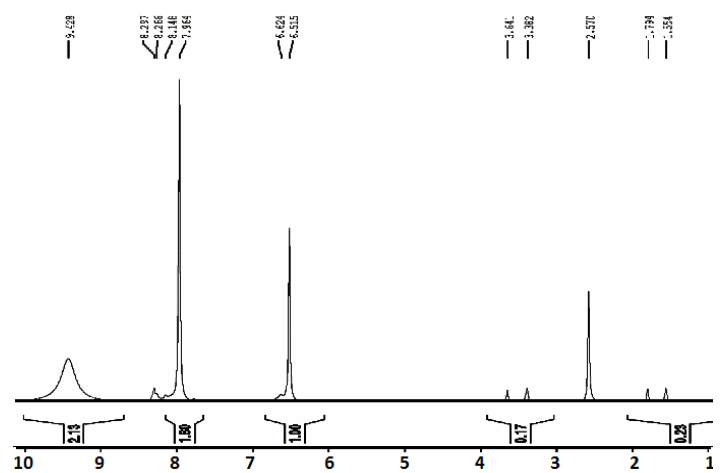

Fig. 7. ${ }^{1} \mathrm{H}-\mathrm{NMR}$ of $\mathrm{Mo}_{2} \mathrm{O}_{4} \mathrm{Cl}_{4}\left(\mathrm{C}_{3} \mathrm{H}_{4} \mathrm{~N}_{2}\right)_{4}$, [7]

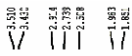

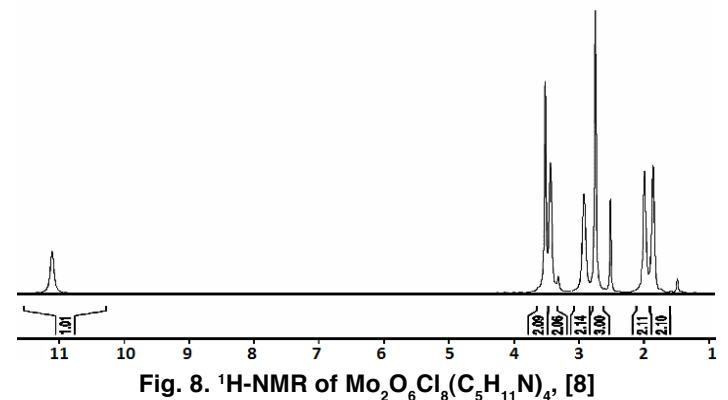

${ }^{13} \mathrm{C}$ NMR Spectra

Spectra were taken in DMSO- $d_{6}$ solvent. Solvent residual peak of DMSO- $d_{6}{ }^{80}$ occurs at $39.52 \pm 0.06 \mathrm{ppm}$. THF ${ }^{87}$ spectrum in DMSO-d ${ }_{6}$ shows $\mathrm{O}-\mathrm{CH}_{2}$ peak and $\mathrm{CH}_{2}$ peak at 67.03 ppm and $25.14 \mathrm{ppm}$, respectively. In the spectra given below, $\uparrow$ and $\downarrow$ represent upfield/ downfield shift.

Spectrum of [3] shows that there is slight upfield shift of all absorptions of 1,4-diaminobutane ${ }^{85}$. This may be due to change of solvent from $\mathrm{CDCl}_{3}$ to DMSO-d $_{6}$ (Fig. 9, Table 14). 
Table 14: $\left({ }^{13} \mathrm{C}\right.$ NMR absorptions in ppm)

\begin{tabular}{lcc}
\hline Assignments & 1,4-Diaminobutane ${ }^{85}{\text { in } \mathrm{CDCl}_{3}}$ & {$[3]$} \\
\hline C attached to nitrogen & 41 & $38.24 \uparrow$ \\
Other C & 29 & $25.04 \uparrow$ \\
Residual $^{80}$ DMSO-d $_{6}$ & & 39.50 \\
\hline
\end{tabular}

Spectrum of [4] shows that there is slight upfield shift of C-2 and C-6 of 3-methylpyridine ${ }^{86}$, whereas there is slight downward shift of C-3, C-4 and $\mathrm{C}-5$. This is due to flow of $\pi$-electron density from C-3, C-4 and C-5 to N through C-2 and C-6, when $\mathrm{N}$ coordinates with Mo (Fig. 10, Table 15).

Table 15: ( ${ }^{13} \mathrm{C}$ NMR absorptions in ppm)

\begin{tabular}{|c|c|c|}
\hline Assignments & 3-Methylpyridine ${ }^{86}$ in $\mathrm{CDCl}_{3}$ & [4] \\
\hline $\mathrm{C}-2$ (attached to $\mathrm{CH}_{3}$ ) & 150.27 & $146.14 \uparrow$ \\
\hline $\mathrm{C}-3$ & 133.08 & $136.27 \downarrow$ \\
\hline C-4 & 136.40 & $138.77 \downarrow$ \\
\hline C-5 & 123.16 & $126.43 \downarrow$ \\
\hline C-6 & 146.93 & $140.83 \uparrow$ \\
\hline & 18.36 & $17.94 \uparrow$ \\
\hline Residual $80{ }^{3}$ DMSO- $d_{6}$ & & 39.42 \\
\hline $\mathrm{THF}^{87} \mathrm{C}-2,5$ (attached to $\mathrm{N}$ ) & & 65.88 \\
\hline $\mathrm{THF}^{87} \mathrm{C}-3,4$ & & 23.97 \\
\hline
\end{tabular}

Spectrum of [7] shows that there is practically no change in chemical shift of pyrazole ${ }^{88}$ on $\mathrm{N} \rightarrow$ Mo coordination (Fig. 11, Table 16).

Table 16: $\left({ }^{13} \mathrm{C}\right.$ NMR absorptions in ppm)

\begin{tabular}{lcc}
\hline Assignments & Pyrazole $^{88}$ in $\mathrm{CDCl}_{3}$ & [7] \\
\hline Carbons attached to N atoms & 134.0 & $133.12 \uparrow$ \\
Remaining carbona & 105.1 & $104.81 \uparrow$ \\
Residual $^{80}$ DMSO-d $_{6}$ & & 39.42 \\
\hline
\end{tabular}

Spectrum of [8] shows that there is slight upfield shift of all absorptions of 1-methylpyrrolidine ${ }^{89}$. This may be due to change of solvent from $\mathrm{CDCl}_{3}$ to DMSO-d $_{6}$ (Fig. 12, Table 17).

Table 17: ( ${ }^{13} \mathrm{C}$ NMR absorptions in ppm)

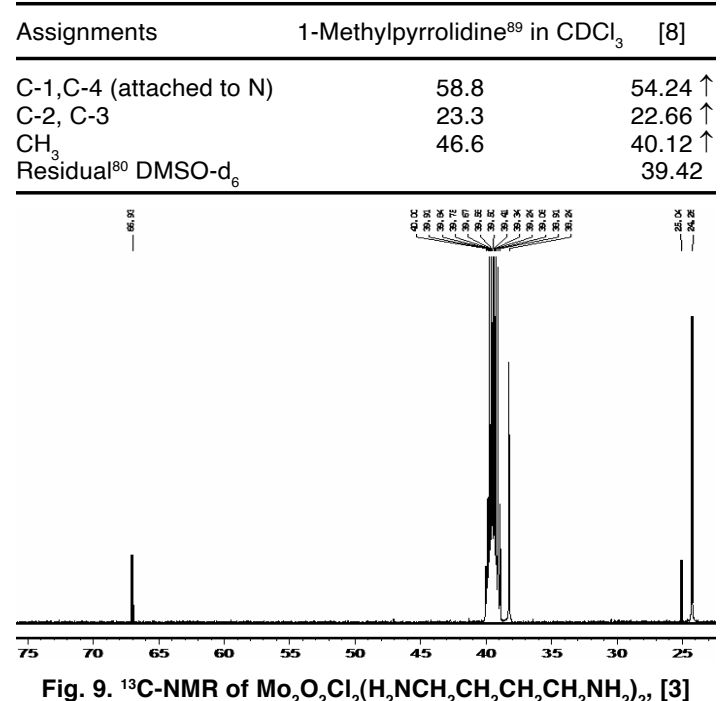

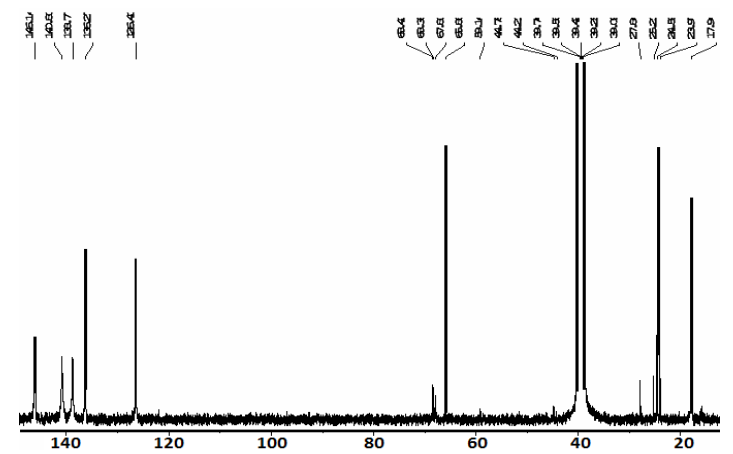

Fig. 10. ${ }^{13} \mathrm{C}-\mathrm{NMR}$ of $\mathrm{Mo}_{3} \mathrm{Cl}_{8}\left(\mathrm{C}_{6} \mathrm{H}_{7} \mathrm{~N}\right)_{4}\left(\mathrm{C}_{4} \mathrm{H}_{8} \mathrm{O}\right)_{2}$, [4]
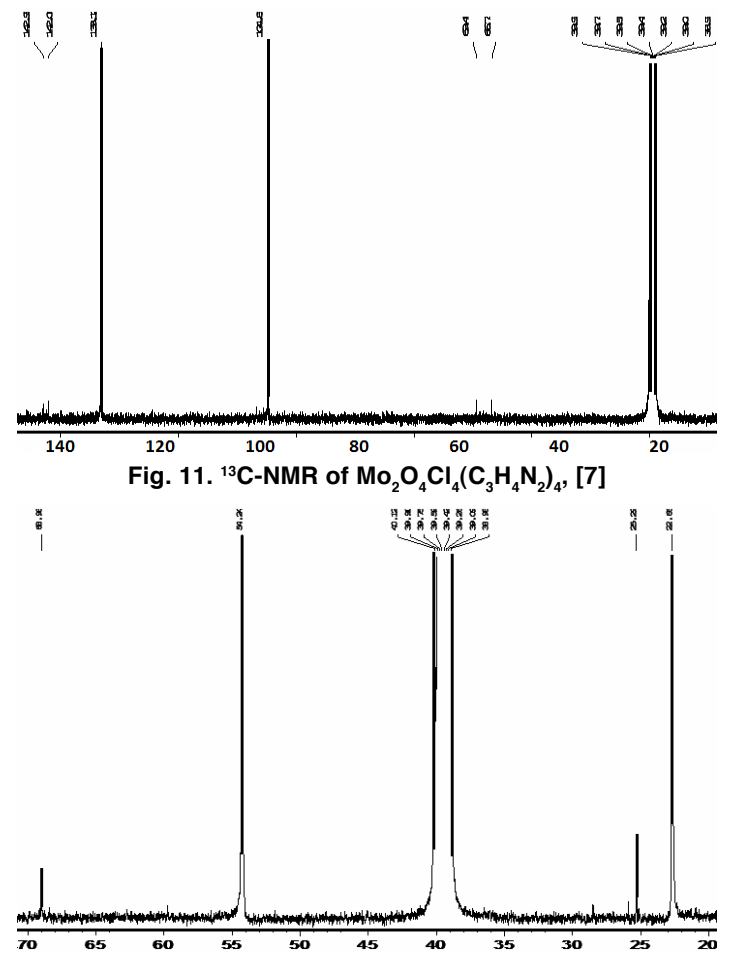

Fig. 12. ${ }^{13} \mathrm{C}$-NMR of $\mathrm{Mo}_{2} \mathrm{O}_{6} \mathrm{Cl}_{8}\left(\mathrm{C}_{5} \mathrm{H}_{11} \mathrm{~N}\right)_{4}$, [8]

Microbiological Activity

Molybdenum compounds prepared were tested using agar well diffusion assay method for their antibacterial and antifungal potential on the strains: Staphylococcus aureus (Gram-positive bacteria) (MTCC-737), E. coli (Gram-negative bacteria) (MTCC-1687), Candida albicans (fungus) (MTCC-227) and Aspergillus niger (fungus) (MTCC282). Standard drugs amoxicillin and ketoconazole were used for bacteria and virus, respectively as reference. Zone of inhibition ${ }^{90}$ for a strain of bacteria/ fungi was estimated to ascertain the amount of resistance of bacteria/fungi to the drug used as reference. Molybdenum compounds synthesized have been noted as potentially active against the above said bacteria and fungi (Table 18). Especially, 
1. Compounds $1,2,4,5$ and 8 have greater antibacterial activity against $E$. coli than the reference drug (amoxicillin).

2. Compounds 1,2 and 5 have greater antifungal activity against $C$. albicans than the reference drug (ketoconazole).

\begin{tabular}{|c|c|c|c|c|}
\hline \multirow[t]{3}{*}{ Compound (100 $\mu \mathrm{g} / \mathrm{mL})$} & \multicolumn{3}{|c|}{ Zone of inhibition ${ }^{90}(\mathrm{~mm})$} & \\
\hline & Gram-positive & Gram-negative & \multicolumn{2}{|c|}{ Antifungal } \\
\hline & S. aureus & E. coli & C. albicans & A. niger \\
\hline Reference Drug & 25.69 & 18.35 & 21.37 & 28.21 \\
\hline [1] & 24.12 & 19.56 & 21.74 & 21.56 \\
\hline [2] & 19.28 & 22.51 & 23.12 & 21.58 \\
\hline$[4]$ & 19.84 & 22.21 & 19.52 & 21.69 \\
\hline [5] & 21.54 & 49.62 & 21.47 & 19.87 \\
\hline [8] & 23.11 & 22.61 & 19.85 & 18.72 \\
\hline
\end{tabular}

Conclusion and results: These compounds can kill and inhibit the growth of microbes

Table 19: (LC-MS Ionization)

\section{Mass Spectra (LC-MS)}

Theoretical $\mathrm{m} / \mathrm{z}$ values of the fragments have been calculated ${ }^{91}$ on the basis of the most abundant isotopes of the individual elements. Fragments detected (Tables 19,20) reinforce the formulae,

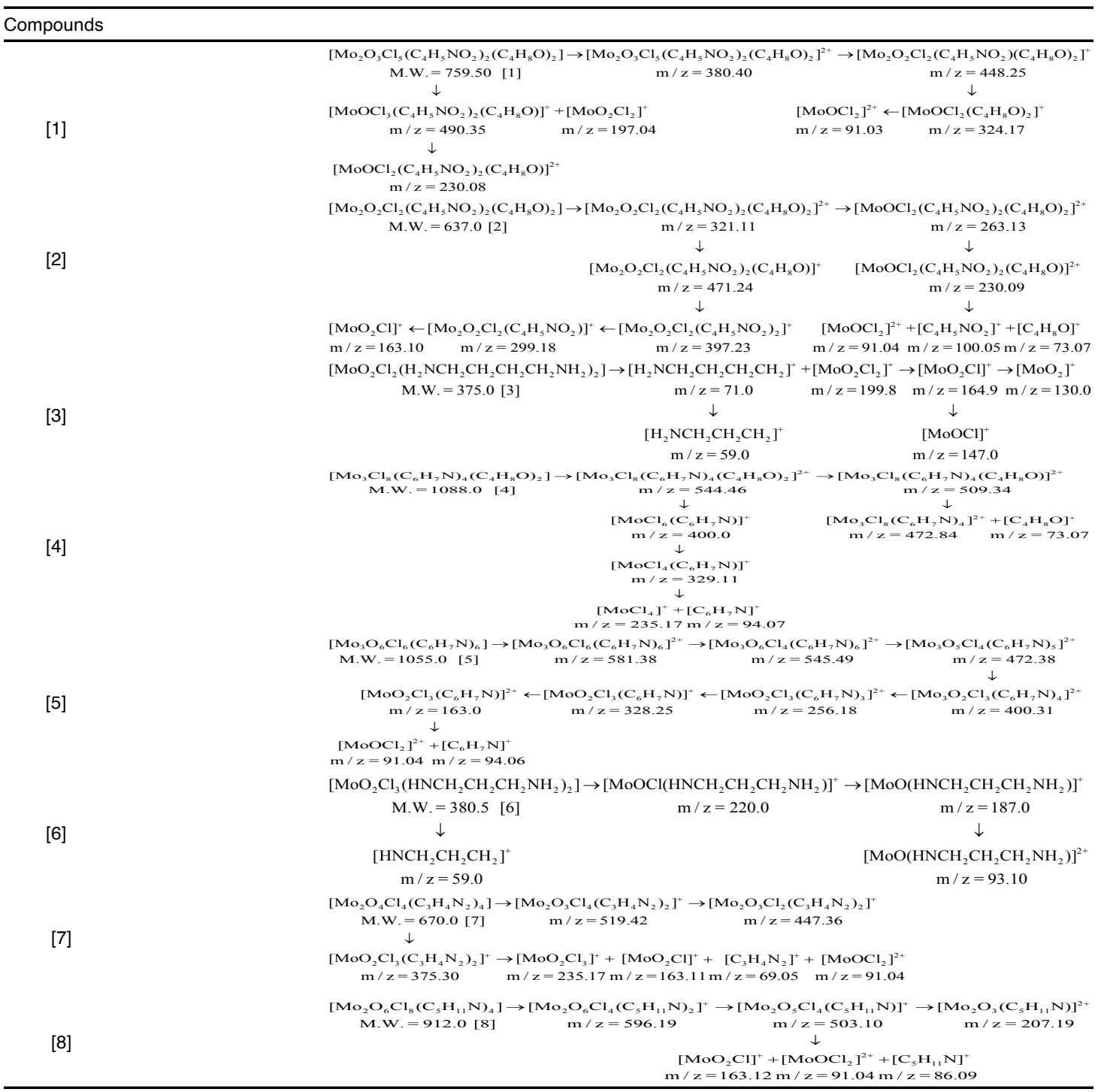


SINGH, KUMAR., Orient. J. Chem., Vol. 37(3), 553-567 (2021)

Table 20: (LC-MS Ion $\mathrm{m} / \mathrm{z}$ values)

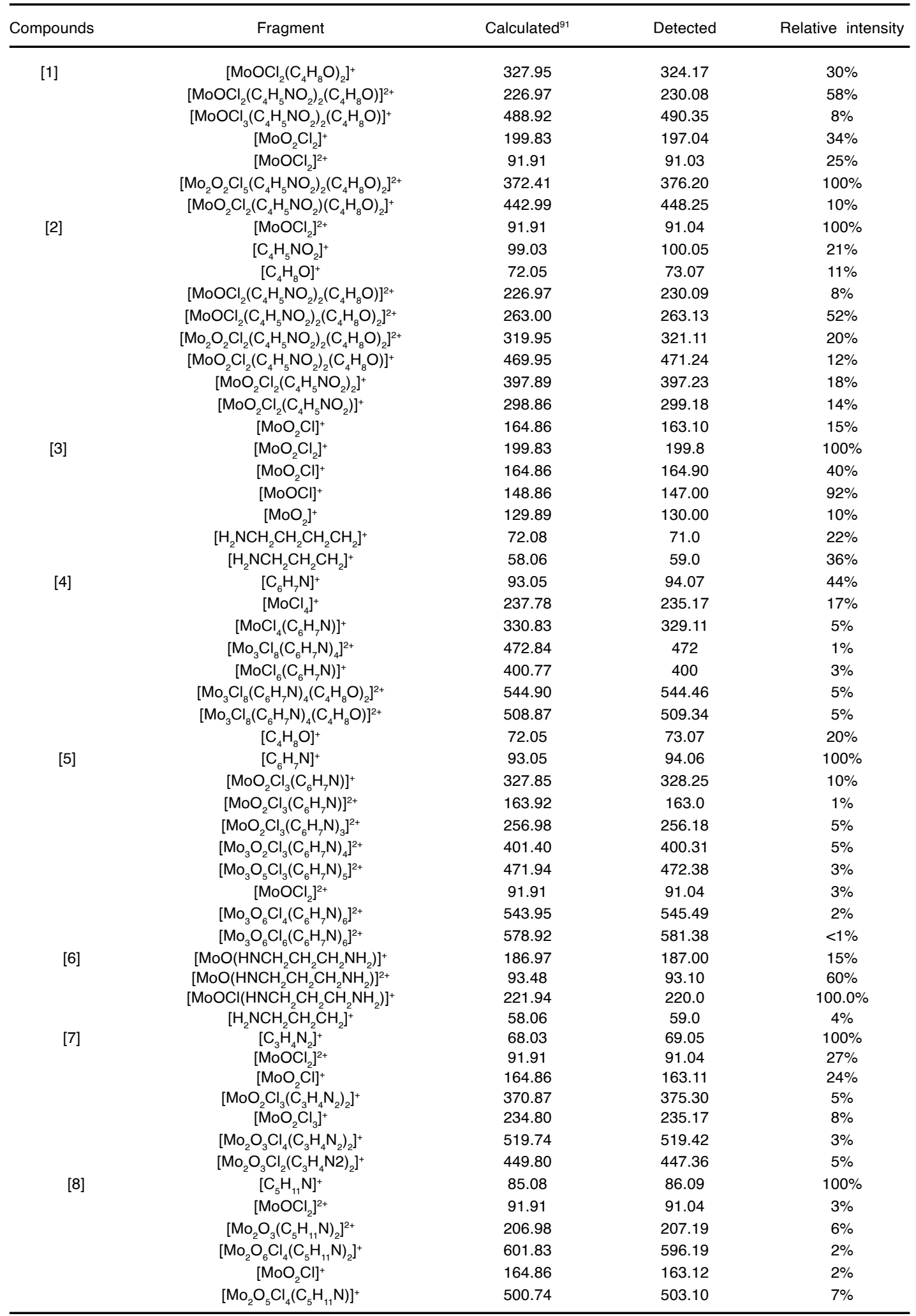




\section{CONCLUSION}

Band at $3294 \mathrm{~cm}^{-1}$ indicates that [1] contains succinimide $\mathrm{N}-\mathrm{H}$ group. Bands at 980 $\mathrm{cm}^{-1}$ and $919 \mathrm{~cm}^{-1}$ support the availability of cis$\mathrm{MoO}_{2}{ }^{2+}$ core in [1]. Occurrence of cis- $\mathrm{MoO}_{2}{ }^{2+}$ core is due to oxo abstraction by molybdenum from THF. There is decrease in $\mathrm{C}=\mathrm{O}$ sym and asym absorptions due to decrease in $\mathrm{C}=\mathrm{O}$ bond order on $\mathrm{O} \rightarrow$ Mo coordination in [1]. Succinimide $\mathrm{CH}_{2}$ absorb at 2.73 ppm. Spectrum of [1] shows $\mathrm{CH}_{2}$ absorption at $3.63 \mathrm{ppm}$ showing downfield shift due to decrease in electron density around these protons on coordination with molybdenum through carbonyl group. Microbiological studies reveal that [1] is effective against the bacteria/fungi tested for, especially $E$. coli and $C$. albicans, where [1] is more effective than the reference drugs themselves. Elemental analysis and LC-MS fragmentation support the proposed formula.

Band at $3433 \mathrm{~cm}^{-1}$ indicates that [2] contains succinimide $\mathrm{N}-\mathrm{H}$ group. Bands at $984 \mathrm{~cm}^{-1}$ and $923 \mathrm{~cm}^{-1}$ support the availability of cis- $\mathrm{MoO}_{2}{ }^{2+}$ core in [2]. Occurrence of cis- $\mathrm{MoO}_{2}{ }^{2+}$ core is due to oxo abstraction by molybdenum from THF. There is decrease in $\mathrm{C}=\mathrm{O}$ sym and asym absorptions due to decrease in $\mathrm{C}=\mathrm{O}$ bond order on $\mathrm{O} \rightarrow \mathrm{Mo}$ coordination in [2]. Spectrum of [2] shows $\mathrm{CH}_{2}$ absorption at $3.42 \mathrm{ppm}$ showing downfield shift due to decrease in electron density around these protons on coordination with molybdenum through carbonyl group. Microbiological studies reveal that [2] is effective against the bacteria/fungi tested for, especially $E$. coli and $C$. albicans, where [2] is more effective than the reference drugs themselves. Elemental analysis and LC-MS fragmentation support the proposed formula.

Strong 1,4-diaminobutane N-H absorptions have been recorded at $3391 \mathrm{~cm}^{-1}, 3077 \mathrm{~cm}^{-1}$ and $3010 \mathrm{~cm}^{-1}$ in [3]. Terminal $\mathrm{Mo}=\mathrm{O}$ stretching occurs at $990 \mathrm{~cm}^{-1}-1010 \mathrm{~cm}^{-1}$ in various inert solvents. A medium $\mathrm{Mo}=\mathrm{O}$ stretching at $921 \mathrm{~cm}^{-1}$ conforms to the presence of terminal $\mathrm{Mo}=\mathrm{O}$ group. There is a decline in $\mathrm{Mo}=\mathrm{O}$ stretching to $921 \mathrm{~cm}^{-1}$ showing Mo coordination to 1,4-diaminobutane through $\mathrm{N}$ atom, in a direction trans to $\mathrm{Mo}=\mathrm{O}$ bond. Bending mode due to $\mathrm{NH}_{2}$ observed in 1,4-diaminobutane at 1146 $\mathrm{cm}^{-1}$ is declined to $1116 \mathrm{~cm}^{-1}$, because of $\mathrm{N} \rightarrow \mathrm{Mo}$ coordination. 1, 4-Diaminobutane shows $\mathrm{N}-\mathrm{H}$ peak at $1.15 \mathrm{ppm}$. NMR of [3] suggests that $\mathrm{NH}_{2}$ peak has shifted downfield. Peak of side $\mathrm{CH}_{2}$ (attached to $\mathrm{N}$ which coordinates) as well as peak of middle $\mathrm{CH}_{2}$ (attached to outer $\mathrm{CH}_{2}$ on the side in which $\mathrm{N}$ coordinates) have shifted down field due to decrease in electron density around these protons on $\mathrm{N} \rightarrow \mathrm{Mo}$ coordination. ${ }^{13} \mathrm{C}$ NMR spectrum of [3] shows that there is slight upfield shift of all absorptions of 1,4-diaminobutane. This may be due to change of solvent from $\mathrm{CDCl}_{3}$ to DMSO- $\mathrm{d}_{6}$. Elemental analysis and LC-MS fragmentation support the proposed formula.

Strong bands at $3119 \mathrm{~cm}^{-1}$ and $3054 \mathrm{~cm}^{-1}$ have been noticed in [4] which show presence of 3-methylpyridine ring $\mathrm{C}-\mathrm{H}$ absorptions. Ring $\mathrm{C}=\mathrm{N}$ stretching \& ring $\mathrm{C}=\mathrm{N}$ torsion wave numbers have increased and ring $\mathrm{C}-\mathrm{H}$ bending mode wave numbers have declined due to $\mathrm{Mo}(\mathrm{d} \pi) \rightarrow \mathrm{N}(\mathrm{p} \pi)$ back bonding. A strong band at $989 \mathrm{~cm}^{-1}$ reveals the presence of terminal $\mathrm{Mo}=\mathrm{O}$ in [4]. Comparison of 3-methylpyridine spectrum with that of [4], shows that there is downfield shift for all protons. This is due to reduction in ring $\pi$-electron density around these protons on sharing of lone pair by nitrogen with molybdenum. ${ }^{13} \mathrm{C}$ NMR spectrum of [4] shows that there is slight upfield shift of C-2 and C- 6 of 3-methylpyridine, whereas there is slight downward shift of C-3, C-4 and C-5. This is due to flow of $\pi$-electron density from $\mathrm{C}-3, \mathrm{C}-4$ and $\mathrm{C}-5$ to $\mathrm{N}$, through C-2 and C-6, when N coordinates with Mo. Microbiological studies reveal that [4] is effective against the bacteria/fungi tested for, especially $E$. coli where [4] is more effective than the reference drug itself. Elemental analysis and LC-MS fragmentation support the proposed formula.

Strong band at $3391 \mathrm{~cm}^{-1}$ has been noticed in [5] which shows presence of 3-methylpyridine ring $\mathrm{C}-\mathrm{H}$ absorption. Ring $\mathrm{C}=\mathrm{N}$ stretching \& ring $\mathrm{C}=\mathrm{N}$ torsion wave numbers have increased and ring $\mathrm{C}-\mathrm{H}$ bending mode wave numbers have declined due to $\mathrm{Mo}(\mathrm{d} \pi) \rightarrow \mathrm{N}(\mathrm{p} \pi)$ back bonding. A medium band at 949 $\mathrm{cm}^{-1}$ reveals the presence of terminal $\mathrm{Mo}=\mathrm{O}$ group in [5]. Comparison of 3-methylpyridine spectrum with that of [5], shows that there is downfield shift for all protons. This is due to reduction in ring $\pi$-electron density around these protons on sharing of lone pair by nitrogen with molybdenum. Microbiological studies reveal that [5] is effective against the bacteria/fungi tested for, especially $E$. coli and 
C. albicans, where [5] is more effective than the reference drugs themselves. Elemental analysis and LC-MS fragmentation support the proposed formula.

Absorptions at $3427 \mathrm{~cm}^{-1} \& 3014 \mathrm{~cm}^{-1}$ in [6] suggest the presence of 1,3-diaminopropane $\mathrm{N}-\mathrm{H}$ group in the compound. A strong band at $944 \mathrm{~cm}^{-1}$ is attributed to terminal $\mathrm{Mo}=\mathrm{O}$ stretching. $\mathrm{NH}_{2}$ bending absorption in the range of $1159 \mathrm{~cm}^{-1}-1182 \mathrm{~cm}^{-1}$ is also shifted to lower wave number $1108 \mathrm{~cm}^{-1}$, mainly due to coordination with molybdenum. Occurrence of terminal $\mathrm{Mo}=\mathrm{O}$ is due to oxo abstraction by molybdenum from THF. Comparison of spectrum of 1,3-diaminopropane with that of [6] suggests that $\mathrm{NH}_{2}$ and $\mathrm{CH}_{2}$ absorptions of 1,3-diaminopropane have downfield shift. This is because of decrease in electron density around these protons on $\mathrm{N} \rightarrow \mathrm{Mo}$ coordination. Elemental analysis and LC-MS fragmentation support the proposed formula.

Band at $3386 \mathrm{~cm}^{-1}$ reflects that [7] contains pyrazole $\mathrm{N}-\mathrm{H}$ group. $\mathrm{N}-\mathrm{H}$ stretching is declined due to Mo-N coordination. Medium band at 969 $\mathrm{cm}^{-1}$ shows the existence of terminal $\mathrm{Mo}=\mathrm{O}$ in [7]. Occurrence of terminal $\mathrm{Mo}=\mathrm{O}$ is due to oxo abstraction by molybdenum from THF. Spectrum of pyrazole shows absorptions due to middle $\mathrm{C}-\mathrm{H}$ proton at $6.31 \mathrm{ppm}, \mathrm{C}-\mathrm{H}$ protons on other two carbons at $7.61 \mathrm{ppm}$ and due to $\mathrm{N}-\mathrm{H}$ proton at 12.64 ppm. Spectrum of [7] shows that all the pyrazole $\mathrm{CH}$ protons have moved downfield. This is because of decrease in electron density around these protons on $\mathrm{N} \rightarrow$ Mo coordination. Due to keto-enol tautomerization equilibrium, peaks of $\mathrm{CH}$ protons of pyrazole appear as singlets. ${ }^{13} \mathrm{C}$ NMR spectrum of [7] shows that there is practically no change in chemical shift of pyrazole. Elemental analysis and LC-MS fragmentation support the proposed formula.

Absorption at $2750 \mathrm{~cm}^{-1}$ in [8] shows presence of 1-methylpyrrolidine $\mathrm{C}-\mathrm{H}$ asymmetric the stretching. Weak band corresponding to the presence of terminal $\mathrm{Mo}=\mathrm{O}$ is observed at $976 \mathrm{~cm}^{-1}$. A peak at $3410 \mathrm{~cm}^{-1}$ may be due to 1-methylpyrrolidinium cation. Occurrence of terminal $\mathrm{Mo}=\mathrm{O}$ is due to oxo abstraction by molybdenum from THF. Spectrum of [8] shows that all the $\mathrm{CH}$ absorptions of 1-methylpyrrolidine have moved downfield, referring to decline in electron density of the ring on $\mathrm{N} \rightarrow \mathrm{Mo}$ coordination. There is a broad peak at $11.10 \mathrm{ppm}$ indicating formation of 1-methylpyrrolidinium ion. ${ }^{13} \mathrm{C}$ NMR spectrum of [8] shows that there is slight upfield shift of all absorptions of 1-methylpyrrolidine. This may be due to change of solvent from $\mathrm{CDCl}_{3}$ to DMSO- $d_{6}$. Microbiological studies reveal that [8] is effective against the bacteria/fungi tested for, especially $E$. coli where [8] is more effective than the reference drug itself. Elemental analysis and LC-MS fragmentation support the proposed formula.

\section{ACKNOWLEDGEMENT}

We, the authors thank P. U. Chandigarh, India for providing facility of elemental analysis and spectral studies. We are also thankful to ISF Analytical Laboratory (ISF College of Pharmacy), Ferozepur Road, Moga, Punjab (India) for carrying out microbiological studies.

\section{Conflict of interest}

There is no conflict of interest among the authors.

\section{REFERENCES}

1. Shetgiri, N. P.; Nayak, B. K., Indian J. Chem., 2005, 44B, 1933-1936.

2. Aeberli, P.; Go gerty, J. H.; Houlihan, W. J.; lorio, L. C., J. Med. Chem., 1976, 19(3), 436-438

3. Pennington, F. C.; Guercio, P. A.; Solomons, I. A., J. Amer. Chem. Soc., 1953, 75(9), 2261.

4. Hall, I. H.; Wong, O. T.; Scovill, J. P., Biomed Pharmacother., 1995, 49(5), 251-258.

5. Crider, A. M; Kolczynski, T. M.; Yates, K. M., J. Med. Chem., 1980, 23(3), 324-326.

6. Johnston, T. P.; Piper, J. R.; Stringfellow, C. R., J. Med. Chem., 1971, 14(4), 350-354.

7. Kaczorowski, G. J.; McManus, O. B.; Priest, B. T.; Garcia, M. L., Gen. Physiology., 2008,
131(5), 399-405.

8. Musso, D. L.; Cochran, F. R.; Kelley, J. L.; McLean, E. W.; Selph, J. L.; Rigdon, G. C., J. Med. Chem., 2003, 46(3), 399-408.

9. Rich, D. H.; Gardner, J. H., Tetrahedron Letters., 1983, 24(48), 5305-5308.

10. Zentz, F.; Valla, A.; Guillou, R. L.; Labia, R.; Mathot, A. G.; Sirot, D., Farmaco., 2002, 57(5), 421-426.

11. Correa, R.; Filho, V. C.; Rosa, P. W.; Pereira, C. I.; Schlemper, V.; Nunes, R. J., Pharm. Pharmacol. Comm., 1997, 3(2), 67-71.

12. Kornet, M. J.; Crider, A. M.; Magarian, E. O., J. Med. Chem., 1977, 20(3), 405-409. 
13. Isaka, M.; Prathumpai, W.; Wongsa, P.; Tanticharoen, M.; Hirsutellone, F., Org. Lett., 2006, 8(13), 2815-2817.

14. Filho, V. C.; Nunes, R. J.; Calixto, J. B.; Yunes, R. A., Pharm. Pharmacol. Comm., 1995, 1(8), 399-401.

15. Hazra, B. G.; Pore, V. S.; Day, S. K.; Datta, S.; Darokar, M. P., Saikia, D., Bioorg. Med. Chem. Lett., 2004, 14(3), 773-777.

16. Amah, K. U.; Sylvain, A. Y. G.; Gaston, K. A.; Alice, K. H. M. T.; Baptiste, M. J., International Research Journal of Pure \& Applied Chemistry., 2016, 12(4), 1-11.

17. Wang, X.; Gao, S.; Wang, J.; Xu, S.; Li, H.; Chen, K.; Ouyang, P., Chinese Journal of Chemical Engineering., 2021, 30, 4-13.

18. Gaymans, R. J.; Utteren, T. E. C.; van den Berg, J. W. A.; Schuyer, J., Journal of Polymer Science: Polymer Chemistry Edition., 1977, 15(3), 537-545.

19. Pasupathy, A.; Nirmala, S.; Abirami, G.; Satish, A.; Milton, R. P., International Journal of Scientific and Research Publications., 2014, 4(3), 1-3.

20. Stellman, J. M., Encyclopaedia of Occupational Health and Safety, $4^{\text {th }}$ Edition, International Labour Office, Geneva., 1998, 4.

21. Shimizu, S.; Watanabe, N.; Kataoka, T.; Shoji, T.; Abe, N.; Morishita, S.; Ichimura. H., Ullmann's Encyclopedia of Industrial Chemistry., 2002, doi:10.1002/14356007.a22_399.

22. Sims, G. K.; Sommers, L. E., Environmental Toxicology and Chemistry., 1986, 5, 503-509.

23. Sims, G. K.; Sommers, L. E., J. Environmental Quality., 1985, 14, 580-584.

24. Srinivasu, J. V.; Narendra, K.; Rao, B. S., Indian Journal of Pure \& Applied Physics., 2017, 55, 797-805.

25. Bingham, E.; Cohrssen, B., Patty' Toxicology, John Wiley \& Sons, $6^{\text {th }}$ Edition., 2012, 1.

26. Chae, T. U.; Kim, W. J.; Choi, S.; Park, S. J.; Lee, S. Y., Scientific Reports., 2015, 5, article 23040, 1-13.

27. Abebe, A.; Bayeh, Y.; Belay, M.; Gebretsadik, T.; Thomas, M.; Linert, W., Future Journal of Pharmaceutical Sciences., 2020, 6, Article (13), 1-9.

28. Salim, E. I.; Wanibuchi, H.; Morimura, K.; Kim, S.; Yano, Y.; Yamamoto, S.; Fukushima S., Carcinogenesis., 2000, 21(2), 195-203.

29. J. E. Seely; Pegg, A. E., Biochem. J., 1983, 216, 701-707.

30. Barcelo, M.; Ravina, E.; Masaguer, C. F.; Dominguez, E.; Areias, F. M.; Brea, J., Bioorg. Med. Chem. Lett., 2007, 17, 4873-4877.
31. Pospisil, P.; Folkers, G.; FABAD J. Pharm. Sci., 2004, 29, 81-92.

32. Cho, A. E.; Guallar, V.; Berne, B. J.; Friesner, R., J. Computchem., 2005, 26, 915-931.

33. Bekhit, A. A.; Ashour, H. M. A.; Ghang, Y. S. A.; Bekhit, A. E. A.; Baraka, A., Eur. J. Med. Chem., 2008, 43, 456-463.

34. Aziz, M. A.; Abuorahma, G. E. A.; Hassan, A. A., Eur. J. Med. Chem., 2009, 44, 3480-3487.

35. Ahmed, O. M.; Muhamed, M. A.; Ahmed, R. R.; Ahmed, S. A., Eur. J. Med. Chem., 2009, 44, 3519-3523.

36. Frigola, J.; Colombo, A.; Pares, J.; Martinez, L.; Sagarra, R.; Rosert, R., Eur. J. Med. Chem., 1989, 24, 435-445.

37. Bondock, S.; Rabie, R.; Etman, H. A.; Fadda, A. A., Eur. J. Med. Chem., 2008, 43, 2122-2229.

38. Castagnolo, D.; Mantti, F.; Radi, M.; Bechi, B.; Pagano, M.; Logu, A. D., Bioorg. Med. Chem., 2009, 17, 5716-5721.

39. Gopalakrishnan, S.; Ravi, T. K.; Manojkumar, P., Eur. J. Med. Chem., 2009, 44, 4690-4694.

40. Bekhit, A. A.; Aziem, T. A., Bioorg. Med. Chem., 2004, 12, 1935-1945.

41. Richardson, C. H.; Shepard, H. H., Journal of Agricultural Research., 1930, 40(11), 1007-1015.

42. Page, N.; Stevenson, R.; Powell, M., Analytical Methods., 2014, 6(4), 1248-1253.

43. Abebe, A.; Hailemariam, T., Bioinorganic Chemistry and Applications., 2016, Article ID 3607924, 1-9.

44. Thomas, D. D.; Ridnour, L. A.; Isenberg, J. S.; Flores, S. W.; Switzer, C. H.; Donzelli, S.; Hussain, P.; Vecoli, C.; Paolocci, N.; Ambs, S.; Colton, C. A.; Harris, C. C.; Roberts, D. D.; Wink, D. A., Free Radical Biology and Medicine., 2008, 45(1), 18-31.

45. Chen, P. R.; He, C., Current Opinion in Chemical Biology., 2008, 12(2), 214-21.

46. Pennella, M. A.; Giedroc, D. P., Biometals., 2005, 18(4), 413-28.

47. Cowan, J. A.; Bertini, I.; Gray, H. B.; Stiefel, E. I.; Valentine, J. S., Structure and Reactivity: Biological Inorganic Chemistry, 3, University Science Books, Sausalito, 2007, 8(2): 175181.

48. Jameel, A.; MSA, S. A. P., Asian Journal of Chemistry., 2010, 22(12), 3422-48.

49. Anupama, B.; Sunuta, M.; Leela, D. S.; Ushaiah; Kumari, C. G., Journal of Fluorescence., 2014, 24(4), 1067-76.

50. Singh, G.; Mangla, V.; Goyal, M.; Singla, K.; Rani, D., American International Journal of Research in Science, Technology, Engineering \& Mathematics., 2014, 8(2), 131-136. 
51. Singh, G.; Mangla, V.; Goyal, M.; Singla, K.; Rani, D., American International Journal of Research in Science, Technology, Engineering \& Mathematics., 2015, 10(4), 299-308.

52. Singh, G.; Mangla, V.; Goyal, M.; Singla, K.; Rani, D., American International Journal of Research in Science, Technology, Engineering \& Mathematics., 2015, 11(2), 158-166.

53. Singh, G.; Mangla, V.; Goyal, M.; Singla, K.; Rani, D.; Kumar, R., American International Journal of Research in Science, Technology, Engineering \& Mathematics., 2016, 16(1), 56-64.

54. Singh, G.; Kumar, R., American International Journal of Research in Science, Technology, Engineering \& Mathematics., 2018, 22(1), 01-08.

55. Rani, D.; Singh, G.; Sharma, S., Orient. J. Chem., 2020, 36(6), 1096-1102.

56. Singh, G.; Mangla, V.; Goyal, M.; Singla, K.; Rani, D., International Congress on Chemical, Biological and Environmental Sciences., 2015, 930-942, May 7-9, Kyoto (Japan).

57. Rani, D.; Singh, G.; Sharma, S., Orient. J. Chem., 2021, 37(1), 46-52.

58. Rani, D.; Singh, G.; Sharma, S., Orient. J. Chem., 2021, 37(2), 459-466.

59. Planinic, P.; Meider, H.; Yeh, H.; Vikic-Topic, D., J. Coord. Chem., 1992, 25, 193-204.

60. Behzadi, K.; Baghlaf, A. O.; Thompson, A., J. Less Common Metals., 1978, 57, 103-110.

61. Vogel, A. I., A Text Book of Quantitative Inorganic Analysis; John Wiley and Sons: New York, (Standard methods)., 1963.

62. Stamboliyska, B. A.; Binev, Y. I.; Radomirska, V. B.; Tsenov, J. A.; Juchnovskiet, I. N., Journal of Molecular Structure., 2000, 516, 237-245.

63. Uno, T.; Machida, K., Bulletin of the Chemical Society of Japan., 1962, 35(2), 276-283.

64. Heyn, B.; Hoffmann; Regina, Z. Chem., 1976, 16, 407.

65. Abramenko, V. L.; Sergienko, V. S.; Churakov, A.V., Russian J. Coord. Chem., 2000, 26(12), 866-871.

66. Ergu“ N. Kasap.; Su“ Leyman.; O" Zceli’K., J. Inclusion Phenomena and Molecular Recognition in Chem., 1997, 28, 259-267.

67. Barraclough, C. G.; Kew, D. J., Australian J. Chem., 1970, 23, 2387-2396.

68. Ward, B. G.; Stafford, F. E., Inorg. Chem., 1968, 7, 2569.

69. Abramenko, V. L.; Sergienko, V. S., Russian J. Inorg. Chem., 2009, 54(13), 2031-2053.

70. Toco'n, I. L.; Woolley, M. S.; Otero, J. C.; Marcos, J. I., Journal of Molecular Structure., 1998, 470, 241-246.

71. Gupta, S. K.; Srivastava, T. S., J. Inorganic and Nuclear Chem., 1970, 32, 1611-1615.

72. Hossain, A. G. M. M.; Ogura, K., Indian J. Chem., 1996, 35A, 373-378.

73. Brewerp, D. G.; Wong, P. T. T.; Sears, M. C., Canadian J. Chem., 1968, 46(20), 3119-3128.

74. Yadav, S.; Moheman, A.; Siddiqi, K. S., Arabian Journal of Chemistry., 2016, 9, suppliment 2, S1747-S1754.

75. Stamboliyska, B. A.; Binev, Y. I.; Radomirska, V. B.; Tsenov, J. A.; Juchnovski, I. N., Journal of Molecular Structure., 2000, 516, 237-245.

76. Uno, T.; Machida, K., Bulletin of the Chemical Society of Japan., 1962, 35(2), 276-283.

77. Hoa, N. V.; Tuan, N. A.; Thao, P. T.; Huyen, T. T. T., Journal of Science and Technology., 2016, 54(2), 231-237.

78. Wang, G. T.; Mui. C.; Tannci, J. F.; Filler, M. A.; Musgrave, C. B.; Bent, S. F., J. Phys. Chem., B., 2003, 107, 4982-4996.

79. Szafran, M.; Koput, J.; Szafran, Z. D.; Kwiatkowski, J. S., Vibrational Spectroscopy., 2000, 23, 1-11.

80. Gottlieb, H. E.: Kotlyar, V.; Nudelman, A., J. Org. Chem., 1997, 62, 7512-7515.

81. Olmo, C.; Casas, M. T.; Martínez, J. C.; Franco, Puiggalí, L.; J., Polymers., 2019, 11(4), 572, 1-19.

82. Kumari, N.; Sharma, M.; Das,, P.; Dutta, D. K., Applied Organomet. Chem., 2002, 16, 258-264.

83. Chatterjee, C.; Phulambrikar, A.; Das, S., J. Coord. Chem., 1990, 21(3), 231-236.

84. Editor: Teresa M. V. D. Pinho e Melo, Recent Research Developments in Heterocyclic Chemistry,: 397-475 ISBN: 81-308-0169-8., 2007.

85. https://spectrabase.com/compound/ GhReRxNt2Lp\#9R7ID3SyH4X.

86. https://www.chemicalbook.com/ SpectrumEN_108-99-6_13CNMR.htm.

87. Babij, Ni. R.; E. O.; McCusker, Whiteker, G. T.; Canturk, B.; Choy, N.; Creemer, L. C.; Amicis, C. V. D.; Hewlett, N. M.; Johnson, P. L.; Knobelsdorf, J. A.; Li, F.; Lorsbach, B. A.; Nugent, B. M.; Ryan, S. J.; Smith, M. R.; Yang, Q., Org. Process Res. Dev., 2016, 20, 661-667.

88. Begtrup, M.; Boyer, G.; Cabildo, P.; Cativiela, C.; Claramunt, R. M.; Elguero, J.; Garcia, J. I.; Toiron, C.; Vedser, P., Magnetic Resonance in Chemistry., 1993, 31, 107-168.

89. http://www.molbase.com/en/hnmr_120-94-5moldata-22594.html.

90. Bhattacharjee, M. K., The Journal of Antibiotics., 2015, 68, 657-659.

91. Audi, G.; Wapstra, A. H., Nucl. Phys. A., 1995, 595, 409-480. 